Homology, Homotopy and Applications, vol.13(1), 2011, pp.319-348

\title{
ČECH APPROXIMATION TO THE BROWN-GERSTEN SPECTRAL SEQUENCE
}

\author{
BENJAMIN ANTIEAU
}

(communicated by Brooke Shipley)

\begin{abstract}
In this paper, we show that the étale index of a torsion cohomological Brauer class is divisible by the period of the class. The tool used to make this computation is the Čech approximation of the title. To create the approximation, we use the folklore theorem that the homotopy limit and Postnikov spectral sequences for a cosimplicial space agree beginning with the $E_{2}$-page. As far we know, this folklore theorem has no proof in the literature, so we include a proof.
\end{abstract}

\section{Introduction}

Let $X$ be a geometrically connected scheme, and let $\operatorname{Br}^{\prime}(X)=\mathrm{H}^{2}\left(X_{\text {ét }}, \mathbb{G}_{m}\right)_{\text {tors }}$ be the cohomological Brauer group of $X$. There are two integer invariants of a Brauer class $\alpha \in \operatorname{Br}^{\prime}(X)$. The first is the period of $\alpha$; it is the order of $\alpha$ in the group $\operatorname{Br}^{\prime}(X)$, and it is generally written as $\operatorname{per}(\alpha)$. The second is the index of $\alpha$. If $\mathcal{A}$ is an Azumaya algebra on $X$, then it has rank $n^{2}$ as a locally free $\mathcal{O}_{X}$-module. The integer $n$ is called the degree of $\mathcal{A}$. For a class $\alpha$, its index $\operatorname{ind}(\alpha)$ is defined as the greatest common divisor of all integers $n$ such that there is an Azumaya algebra $\mathcal{A}$ in the class of $\alpha$ of degree $n$. If no such Azumaya algebra exists, then the index is defined to be $+\infty$.

In general,

$$
\operatorname{per}(\alpha) \mid \operatorname{ind}(\alpha) \text {. }
$$

If $X=\operatorname{Spec} k$ for a field $k$, then the index is finite, and the period and the index have the same prime divisors. Therefore, there is a least integer $e(\alpha)$ such that

$$
\operatorname{ind}(\alpha) \mid(\operatorname{per}(\alpha))^{e(\alpha)} .
$$

As $\alpha$ ranges over all Brauer classes, it is an interesting open question to determine the values that $e(\alpha)$ can take on. In [CT], Colliot-Thélène asks whether the following is true.

This material is based upon work supported by the NSF under Grant No. DMS-0901373.

Received May 25, 2010, revised February 4, 2011, March 6, 2011; published on June 6, 2011. 2000 Mathematics Subject Classification: 14F22, 18G40, 16K50, $19 \mathrm{D} 23$.

Key words and phrases: homotopy spectral sequence, cosimplicial space, Brauer group, twisted sheaf. Article available at http://intlpress . com/HHA/v13/n1/a12 and doi:10.4310/HHA .2011.v13.n1.a12 Copyright (C) 2011, International Press. Permission to copy for private use granted. 
Conjecture 1.1 (Period-Index Conjecture). Let $k$ be a field of dimension $d>0$. Then,

$$
\operatorname{ind}(\alpha) \mid(\operatorname{per}(\alpha))^{d-1}
$$

for all $\alpha \in \operatorname{Br}(k)$.

The notion of dimension in the conjecture is not entirely settled. The conjecture is made specifically $[\mathrm{CT}]$ for function fields of $d$-dimensional varieties over algebraically closed fields, or for $C_{d}$ fields. But it is known to be false for cohomological dimension.

Several cases in low dimensions are known, but the question is open in general. For example, it is not known for any function field $\mathbb{C}(X)$ of an algebraic 3 -fold $X$. The importance of the conjecture for $k(X)$ is that it gives information both about the geometry of $X$ and about the arithmetic of $k(X)$ when $X$ is an algebraic variety over an algebraically closed field $k$.

In $[\mathrm{A}]$, we introduced a new invariant $\operatorname{eti}(\alpha)$ for $\alpha \in \operatorname{Br}^{\prime}(X)$, which we call the étale index. By the definition,

$$
\text { eti }(\alpha) \mid \operatorname{ind}(\alpha) \text {. }
$$

The main theorem of that paper is an analogue of Equation (2) when $X=\operatorname{Spec} k$. Specifically, if $k$ is a field of finite cohomological dimension $d=2 c$ or $d=2 c+1$, then

$$
\text { eti }(\alpha) \mid(\operatorname{per}(\alpha))^{c}
$$

when the prime divisors of $\operatorname{per}(\alpha)$ are "large" with respect to $d$. Specifically, the statement is true when $d<2 q$ for all primes $q$ that divide $\operatorname{per}(\alpha)$.

The main result of this paper, Theorem 8.7, is that the analogue of Equation (1) holds for the étale index as well:

$$
\operatorname{per}(\alpha) \mid \operatorname{eti}(\alpha) .
$$

In particular, it is not equal to 1 if $\alpha$ is non-trivial. Therefore, the étale index possesses similar formal properties to the index. However, the étale index is always finite for schemes $X$ of finite étale cohomological dimension, while certain Brauer classes on non-quasi-projective schemes have infinite index.

Here is an application of Theorem 8.7. Define

$$
\begin{aligned}
\mathbf{K}_{0}^{\alpha}(k)^{(0)} & =\mathbf{K}_{0}^{\alpha}(k) / \operatorname{ker}\left(\operatorname{rank}: \mathbf{K}_{0}^{\alpha}(k) \rightarrow \mathbb{Z}\right) \\
\mathbf{K}_{0}^{\alpha, \text { ét }}(k)^{(0)} & =\mathbf{K}_{0}^{\alpha, \text { ét }}(k) / \operatorname{ker}\left(\operatorname{rank}: \mathbf{K}_{0}^{\alpha, \text { ét }}(k) \rightarrow \mathbb{Z}\right),
\end{aligned}
$$

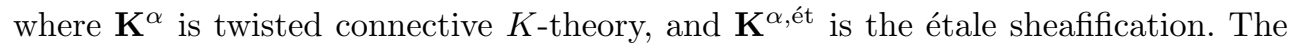
image of the rank on $\mathbf{K}_{0}^{\alpha}(k)$ is generated by $\operatorname{ind}(\alpha)$, while the image of the rank on $\mathbf{K}_{0}^{\alpha, \text { ét }}$ is generated by eti $(\alpha)$. There is an inclusion

$$
\mathbf{K}_{0}^{\alpha}(k)^{(0)} \subseteq \mathbf{K}_{0}^{\alpha, \text { ét }}(k)^{(0)}
$$

with cokernel $F=\mathbb{Z} /\left(\frac{\operatorname{ind}(\alpha)}{\operatorname{eti}(\alpha)}\right)$. In the untwisted case $(\alpha=0)$, the cokernel is always zero. On the other hand, Equation (3), together with the sharpness of some known cases of the period-index conjecture, imply that $F$ is in general not zero when $\alpha \neq 0$.

Suppose that the period-index conjecture of Colliot-Thélène is true. Then, the fact that $\operatorname{per}(\alpha) \mid \operatorname{eti}(\alpha)$ implies that the cokernel $F$ is of order at most $\operatorname{per}(\alpha)^{d-2}$. 
Conversely, if one could prove that the cokernel was of order at most $\operatorname{per}(\alpha)^{c}$ when $d$ is odd or $\operatorname{per}(\alpha)^{c-1}$ when $d$ is even, then Equation (3) would imply the period-index conjecture, at least away from some small primes. Thus, our result allows a translation of the period-index conjecture into a question about the arithmetic failure of étale descent for twisted $K$-theory.

The proof of Theorem 8.7 is based on the computation of a differential in the descent spectral sequence associated to $\mathbf{K}^{\alpha}$. Recall that the descent spectral sequence, or Brown-Gersten spectral sequence, is

$$
\mathrm{E}_{2}^{s, t}=\mathrm{H}^{s}\left(X_{\text {ét }}, \mathcal{K}_{t}^{\alpha}\right) \Rightarrow \mathbf{K}_{t-s}^{\alpha, \text { ét }}(X),
$$

with differentials $d_{r}^{\alpha}$ of degree $(r, r-1)$. In [A], we proved that the twisted $K$-theory sheaves are isomorphic, in a natural way, to the untwisted $K$-theory sheaves. In particular, $\mathcal{K}_{0}^{\alpha} \cong \mathbb{Z}$, and $\mathcal{K}_{1}^{\alpha} \cong \mathbb{G}_{m}$. The étale index is defined to be the least integer $n$ such that $d_{r}^{\alpha}(n)=0, r \geqslant 2$, where we view $n$ as an element in $\mathrm{H}^{0}\left(X, \mathcal{K}_{0}^{\alpha}\right)$. The proof of the main theorem, that $\operatorname{per}(\alpha) \mid \operatorname{eti}(\alpha)$, is given by proving that $d_{2}^{\alpha}(1)=\alpha$, where $\alpha \in \mathrm{H}^{2}\left(X_{\text {ét }}, \mathbb{G}_{m}\right) \cong \mathrm{H}^{2}\left(X_{\text {ét }}, \mathcal{K}_{1}^{\alpha}\right)$. Indeed, once this is done, the least integer such that $d_{2}^{\alpha}(n)=0$ is $n=\operatorname{per}(\alpha)$. This computation is analogous to one given by Kahn and Levine in [KL, Proposition 6.9.1] for the étale motivic spectral sequence converging to étale twisted $K$-theory and another given by Atiyah and Segal in [AS, Proposition 4.6] in the topological twisted case.

To make the computation of the differential, we introduce a Čech approximation to the descent spectral sequence. This in turn relies on the comparison theorem, Theorem 4.7, which says that the homotopy limit and Postnikov spectral sequences for cosimplicial spaces agree beginning with the $\mathrm{E}_{2}$-page. This is a folklore theorem, and we include a proof since we know of no reference for it. Both types of spectral sequences arise frequently in practice in homotopy theory due to the mechanism of cosimplicial replacement of presheaves of spaces, and the theorem easily applies in this situation to say that for a presheaf of spaces on a small category, the homotopy limit and Postnikov spectral sequences agree beginning with the $\mathrm{E}_{2}$-page.

Once this approximation is established, it remains to describe the $d_{2}$ differential for the homotopy limit spectral sequence of a cosimplicial space, and then to translate this description to the Cech approximation spectral sequence.

Some background is given in Sections 2 and 3. Several spectral sequences for cosimplicial spaces are introduced, and a comparison theorem proven, in Section 4. This is applied to presheaves of spectra on a Grothendieck site in Section 5. The differentials of the homotopy limit spectral sequence are described in Section 6 , and those of the Cech approximation in Section 7. The main theorem is proven in Section 8. Finally, in Section 9, the cokernel $F$ of Equation (4) is related in detail to the period-index conjecture.

\section{Acknowledgements}

This paper is part of my Ph.D. dissertation, which was written under the direction of Henri Gillet at UIC. I owe him a great deal of thanks for his support. I have also spoken with Peter Bousfield, David Gepner, Christian Haesemeyer, Jumpei Nogami, and Brooke Shipley about this work. They have all been very helpful and encouraging. 
Additionally, the referee did a very careful job which certainly has resulted in an improvement in the exposition.

\section{Spectral sequences associated to towers of fibrations}

The first quadrant spectral sequences of this section are generalized in the sense that not every term is an abelian group. They have the property $\mathrm{E}_{1}^{s, t}$ is a group when $t-s=1$ and it is a pointed set when $t-s=0$. Nonetheless, there is a good notion of such spectral sequences. For details on these generalized spectral sequences as well as all the other material and notation in this section, see [BK, Section IX.4, esp. 4.1].

\section{Construction 2.1. Let}

$$
\cdots \rightarrow X_{n} \rightarrow X_{n-1} \rightarrow \cdots \rightarrow X_{1} \rightarrow X_{0} \rightarrow *
$$

be a tower of fibrations of pointed spaces. Let $F_{s}$ be the fiber of $X_{s} \rightarrow X_{s-1}$, and denote by $X$ the limit of the tower.

There are long exact sequences associated to the fibrations $F_{s} \rightarrow X_{s} \rightarrow X_{s-1}$ :

$$
\cdots \rightarrow \pi_{t-s} F_{s} \stackrel{k}{\rightarrow} \pi_{t-s} X_{s} \stackrel{i}{\rightarrow} \pi_{t-s} X_{s-1} \stackrel{j}{\rightarrow} \pi_{t-s-1} F_{s} \rightarrow \cdots .
$$

These sequences continue all the way down to $\pi_{0} X_{s-1}$ :

$$
\pi_{2} X_{s-1} \stackrel{j}{\rightarrow} \pi_{1} F_{s} \stackrel{k}{\rightarrow} \pi_{1} X_{s} \stackrel{i}{\rightarrow} \pi_{1} X_{s-1} \stackrel{j}{\rightarrow} \pi_{0} F_{s} \stackrel{k}{\rightarrow} \pi_{0} X_{s} \stackrel{i}{\rightarrow} \pi_{0} X_{s-1}
$$

and

$$
\pi_{1} X_{s-1} \stackrel{j}{\rightarrow} \pi_{0} F_{s}
$$

extends to an action of $\pi_{1} X_{s-1}$ on $\pi_{0} F_{s}$ so that $j$ is the map onto the orbit of the basepoint of $F_{s}$ under the action of $\pi_{1} X_{s-1}$. Besides the usual conditions of ker $=\mathrm{im}$ in the range that this makes sense, the exactness of Equation (5) means

- that the quotient of $\pi_{0} F_{s}$ under this action injects into $\pi_{0} X_{s}$,

- that the cokernel (quotient set) of $\pi_{0} F_{s} \stackrel{k}{\rightarrow} \pi_{0} X_{s}$ injects into $\pi_{0} X_{s-1}$,

- that the stabilizer of the action of $\pi_{1} X_{s-1}$ on $\pi_{0} F_{s}$ at the base-point of $F_{s}$ is the quotient of $\pi_{1} X_{s}$ by the image of $\pi_{1} F_{s} \rightarrow \pi_{1} X_{s}$, and

- that $\pi_{2} X_{s-1}$ maps to the center of $\pi_{1} F_{s}$.

The tower of fibrations and the exact sequences above define an exact couple

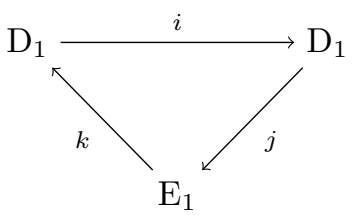


where $D$ and $E$ are bigraded groups:

$$
\begin{aligned}
& \mathrm{D}_{1}^{s, t}=\pi_{t-s} X_{s} \\
& \mathrm{E}_{1}^{s, t}=\pi_{t-s} F_{s} .
\end{aligned}
$$

The maps $i, j$, and $k$ are of bi-degrees $(-1,1),(1,-2)$, and $(0,0)$ :

$$
\begin{aligned}
i: \pi_{t-s} X_{s} & \rightarrow \pi_{t-s} X_{s-1} \\
j: \pi_{t-s} X_{s-1} & \rightarrow \pi_{t-s-1} F_{s} \\
k: \pi_{t-s-1} F_{s} & \rightarrow \pi_{t-s-1} X_{s} .
\end{aligned}
$$

As usual, the exact couple gives rise to a differential $d=j \circ k$ on $E$. It is of bidegree $(1,-2)$. The first derived exact couple is

$$
\begin{gathered}
\pi_{t-s} X_{s}^{(1)}:=\mathrm{D}_{2}^{s t}=\operatorname{im}(i)=\operatorname{im}\left(\pi_{t-s} X_{s+1} \stackrel{i}{\rightarrow} \pi_{t-s} X_{s}\right) \subseteq \pi_{t-s} X_{s} \\
\pi_{t-s} F_{s}^{(1)}:=\mathrm{E}_{2}^{s t}=\mathrm{H}(d)=\operatorname{ker}\left(\pi_{t-s} F_{s} \stackrel{k}{\rightarrow} \pi_{t-s} X_{s} / \operatorname{im}(i)\right) / \\
\operatorname{ker}\left(\pi_{t-s+1} X_{s-1} \stackrel{i}{\rightarrow} \pi_{t-s+1} X_{s-2}\right) .
\end{gathered}
$$

When $s=t$, then the definition of $\mathrm{E}_{2}^{s t}$ should be interpreted as the quotient of the pointed set

$$
\operatorname{ker}\left(\pi_{0} F_{s} \stackrel{k}{\rightarrow} \pi_{0} X_{s} / \operatorname{im}(i)\right)
$$

by the action of $\operatorname{ker}\left(\pi_{1} X_{s-1} \stackrel{i}{\rightarrow} \pi_{1} X_{s-2}\right) \subseteq \pi_{1} X_{s-1}$. Then, the sequences

$$
\pi_{2} X_{s-1}^{(1)} \stackrel{j}{\rightarrow} \pi_{1} F_{s}^{(1)} \stackrel{k}{\rightarrow} \pi_{1} X_{s}^{(1)} \stackrel{i}{\rightarrow} \pi_{1} X_{s-1}^{(1)} \stackrel{j}{\rightarrow} \pi_{0} F_{s}^{(1)} \stackrel{k}{\rightarrow} \pi_{0} X_{s}^{(1)} \stackrel{i}{\rightarrow} \pi_{0} X_{s-1}^{(1)},
$$

are also exact in the generalized sense above.

Repeating this process, one obtains a generalized spectral sequence $\mathrm{E}_{1}^{s, t}\left\{X_{*}\right\}$ associated to the tower, with

$$
\mathrm{E}_{1}^{s, t}=\pi_{t-s} F_{s} \rightarrow \pi_{t-s} X,
$$

where $F_{s}$ is the fiber of $X_{n} \rightarrow X_{n-1}$. The differential $d_{r}$ is of degree $(r, r-1)$. See [BK, Chapter IX]. The harpoon $\rightarrow$ means that the spectral sequence may not converge in the usual sense. Instead, there is a filtration

$$
Q_{s} \pi_{i} X=\operatorname{ker}\left(\pi_{i} X \rightarrow \pi_{i} X_{s}\right)
$$

with successive quotients

$$
e_{\infty}^{s, t}=\operatorname{ker}\left(Q_{s} \pi_{t-s} X \rightarrow Q_{s-1} \pi_{t-s} X\right),
$$

and inclusions

$$
e_{\infty}^{s, t} \subseteq \mathrm{E}_{\infty}^{s, t} .
$$

Write $\mathrm{E}_{1}^{s, t} \Rightarrow \pi_{t-s} X$ when the spectral sequence does hold in the usual sense, in which case equality holds in Equation (6). In this case, say that the spectral sequence converges completely.

There is a second spectral sequence $\tilde{\mathrm{E}}_{2}^{s, t}\left\{X_{*}\right\}$, which is simply a re-indexed version of the first:

$$
\tilde{\mathrm{E}}_{2}^{s, t}=\mathrm{E}_{1}^{t, 2 t-s}=\pi_{t-s} F_{t} \rightarrow \pi_{t-s} X .
$$

This is derived from the exact couple 


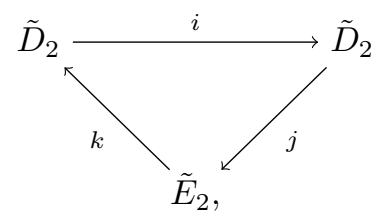

With $\tilde{\mathrm{E}}_{2}$ as above, and

$$
\tilde{\mathrm{D}}_{2}^{s, t}=\pi_{t-s} X_{t} .
$$

The filtration is the same:

$$
\tilde{Q}_{s} \pi_{i} X=Q_{s} \pi_{i} X,
$$

but the successive quotients are

$$
\tilde{e}_{\infty}^{s, t}=e_{\infty}^{t, 2 t-s} .
$$

Here, the differentials are also of degree $(r, r-1)$.

The spectral sequence and the filtration $Q_{s} \pi_{i}$ are functorial for towers of pointed spaces. We view the spectral sequence as including the information of the abutment and the filtration on the abutment. Thus, a morphism of spectral sequences includes a filtration-respecting morphism of the abutment.

Remark 2.2. Under certain conditions, these spectral sequences do converge in some range to some of the homotopy groups of $X$. For instance, suppose that $i \geqslant 1$ and that for each $s \geqslant 0$ there is an integer $N(s) \geqslant 1$ such that

$$
\mathrm{E}_{M}^{s, s+j}=\mathrm{E}_{\infty}^{s, s+j}
$$

for all $M \geqslant N(s)$ when $j=i$ and $j=i+1$. Then, $\mathrm{E}_{*}^{s, t}\left\{X_{*}\right\}$ converges to $\pi_{i} X$. This is also true for $i=0$ when all of the homotopy sets of the spaces in the tower are abelian groups. Again, for details see [BK, Section IX.5].

Remark 2.3. Note that in the application to $K$-theory, the spaces in the tower will have homotopy sets $\pi_{t}$ which are abelian groups for all $t \geqslant 0$. Moreover, the convergence conditions of Equation (7) will always hold under the finite cohomological dimension conditions used in this paper.

\section{Cosimplicial spaces}

Definition 3.1. Let $\Delta$ be the category of finite simplices. Objects of $\Delta$ are nonempty finite ordered sets, and morphisms are set morphisms that preserve order. The category sSets of simplicial sets is the functor category $\operatorname{Fun}\left(\Delta^{\mathrm{op}}\right.$, Sets). In general, if $C$ is a category, then $\mathbf{s} C$ is the category $\operatorname{Fun}\left(\Delta^{\mathrm{op}}, C\right)$, the category of simplicial objects in $C$. Objects of sSets will be called spaces. The category $\mathbf{s S e t s}_{*}$ is the category of pointed spaces.

Definition 3.2. If $C$ is a category, then denote by $\mathbf{c} C$ the functor category $\operatorname{Fun}(\Delta, C)$, the category of cosimplicial objects in $C$. The category of cosimplicial spaces is the category csSets. Write csSets $_{*}$ for the category of cosimplicial pointed spaces. 
Example 3.3. For a space $X$, let the same symbol $X$ denote the constant cosimplicial space $n \mapsto X$.

Example 3.4. The cosimplicial space $\Delta$ is the functor $n \mapsto \Delta^{n}$, where $\Delta^{n}$ is the standard $n$-simplex.

Example 3.5. Let $\mathcal{U}^{\bullet}$ be a hypercover in a site $C$, and let $X$ be a presheaf of simplicial sets on $C$ (a presheaf of spaces). Then, $X_{\mathcal{U}} \bullet$ denotes the cosimplicial space given by evaluating $X$ at each level of $\mathcal{U}^{\bullet}$ in the usual way.

Definition 3.6. If $F$ is an endofunctor of sSets, then one extends $F$ to an endofunctor on csSets by level-wise application. That is, for a cosimplical space $X$, define $F(X)^{n}=F\left(X^{n}\right)$. The typical examples are the $s$-skeleton functors $X \mapsto X[s]$ and the Ex-functor.

Definition 3.7. Let $\mathbf{P}$ be a property of spaces. Then, a cosimplicial space $X$ is level $\mathbf{P}$ if each space $X^{n}$ is $\mathbf{P}$ for $n \geqslant 0$. Similarly, if $\mathbf{Q}$ is a property of morphisms of spaces, then a morphism $f: X \rightarrow Y$ of cosimplicial spaces is level $\mathbf{Q}$ if $f^{n}: X^{n} \rightarrow Y^{n}$ is $\mathbf{Q}$ for all $n \geqslant 0$.

There is a good model structure, the Reedy structure, on cosimplicial spaces. Let $X$ be a cosimplicial space so that $X^{n}$ is a simplicial set for $n \geqslant 0$. A morphism $f: X \rightarrow Y$ is a weak equivalence if each $f^{n}: X^{n} \rightarrow Y^{n}$ is a weak equivalence; that is, if $f$ is a level weak equivalence. The maximal augmentation of a cosimplicial space is the simplicial set that equalizes $d^{0}, d^{1}: X^{0} \rightarrow X^{1}$. A map $f$ of cosimplicial spaces is called a cofibration if it is a level cofibration (level monomorphism) and if it induces an isomorphism on the maximal augmentations. The fibrations are all those morphisms with the right lifting property with respect to acyclic cofibrations. A proof that this is a model category may be found in [BK, Section X.5].

Example 3.8. As examples of cofibrant objects, consider $\Delta$ and $\Delta[s]$. Indeed, $\Delta^{0}$ is a single point, and $\Delta^{1}$ is the 1 -simplex. The coface maps $d^{0}$ and $d^{1}$ send the unique point of $\Delta^{0}$ to the vertices 1 and 0 respectively of $\Delta^{1}$. Therefore, the maximal augmentation is the empty simplicial complex. This also shows that $\Delta[s] \rightarrow \Delta$ is a cofibration.

Let $X$ be a cosimplicial space. Then, define the $n$th matching object of $X$ to be

$$
M^{n} X=\lim _{\phi: \mathbf{n} \rightarrow \mathbf{k}} X^{k},
$$

where $\phi$ runs over all surjections $\mathbf{n} \rightarrow \mathbf{k}$ in $\Delta$. There is a natural map $X^{n+1} \rightarrow M^{n} X$.

Proposition 3.9 ([BK, Section X.4.5]). A morphism $f: X \rightarrow Y$ is a fibration if and only if the induced map

$$
X^{n+1} \rightarrow Y^{n+1} \times_{M^{n} Y} M^{n} X
$$

is a fibration of simplicial sets for all $n \geqslant-1$. 
The closed model structure on cosimplicial spaces is simplicial. That is, there is a functor

$$
\text { Map }: \operatorname{csSets}^{\mathrm{op}} \times \operatorname{csSets} \rightarrow \text { sSets }
$$

defined by

$$
\operatorname{Map}(X, Y): n \mapsto \operatorname{Hom}\left(X \times \Delta^{n}, Y\right)
$$

The space $\operatorname{Map}(X, Y)$ is called the function complex from $X$ to $Y$. Similarly, if $X$ and $Y$ are cosimplicial pointed spaces, then there is a pointed function complex

$$
\operatorname{Map}_{*}(X, Y) \in \mathbf{s S e t s}_{*}
$$

defined by

$$
\operatorname{Map}_{*}(X, Y)_{n}=\operatorname{Hom}\left(X \wedge \Delta_{+}^{n}, Y\right)
$$

Proposition 3.10 ([BK, Section X.5]). The simplicial model category axiom SM7 is satisfied: if $A \rightarrow B$ is a cofibration of cosimplicial spaces and if $X \rightarrow Y$ is a fibration of cosimplicial spaces, then

$$
\operatorname{Map}(B, X) \rightarrow \operatorname{Map}(A, X) \times_{\mathbf{M a p}(A, Y)} \operatorname{Map}(B, Y)
$$

is a fibration.

There is a functor $\mathbf{c s S e t s} \rightarrow \mathbf{c s S e t s}_{*}$ defined by

$$
X=\left(n \mapsto X^{n}\right) \mapsto X_{+}=\left(n \mapsto X_{+}^{n}\right),
$$

where $X_{+}^{n}$ is the space $X^{n}$ with a disjoint basepoint attached.

Definition 3.11. For each integer $n \geqslant 2$, there is a functor

$$
\pi_{n}: \operatorname{csSets}_{*} \rightarrow \mathbf{c A b}
$$

where $\mathbf{c A b}$ is the category of cosimplicial abelian groups, defined by

$$
\pi_{n}(X)^{m}=\pi_{n}\left(X^{m}\right) .
$$

There are also functors, defined by the same equation,

$$
\begin{aligned}
& \pi_{1}: \operatorname{csSets}_{*} \rightarrow \mathbf{c G r o u p s} \\
& \pi_{0}: \operatorname{csSets}_{*} \rightarrow \mathbf{c S e t s}_{*},
\end{aligned}
$$

where $\mathbf{C S e t s}_{*}$ is the category of cosimplicial pointed sets and $\mathbf{c G r o u p s}$ is the category of cosimplicial groups.

Definition 3.12. Let $A$ be a cosimplicial abelian group, cosimplicial group, or cosimplicial pointed set. A pointed cosimplicial space $X$ is called a $K(A, n)$-cosimplicial space if $\pi_{n} X \cong A$, while $\pi_{m} X \cong *$ for $m \neq n$. 


\section{Spectral sequences for cosimplicial spaces}

Let $X$ be a pointed cosimplicial space. Define pointed simplicial complexes

$$
\begin{aligned}
\operatorname{Tot}_{\infty} X & =\operatorname{Map}_{*}\left(\Delta_{+}, X\right), \\
\operatorname{Tot}_{s} X & =\operatorname{Map}_{*}\left(\Delta[s]_{+}, X\right) .
\end{aligned}
$$

By axiom SM7 and Example 3.8, if $X$ is fibrant, then $\operatorname{Tot}_{s} X \rightarrow \operatorname{Tot}_{s-1} X$ gives a tower of pointed fibrations. The inverse limit of this tower is $\operatorname{Tot}_{\infty} X$ when $X$ is fibrant.

Definition 4.1. For an arbitrary cosimplicial pointed space $X$ let $X \rightarrow \mathbb{H}_{c} X$ be a pointed fibrant resolution. Then, the total space spectral sequence of $X,{ }^{\mathbf{T}} \mathrm{E}_{1} X$, is defined to be the spectral sequence of the tower $\operatorname{Tot}_{*} \mathbb{H}_{c} X$ :

$$
{ }^{\mathbf{T}} \mathrm{E}_{1}^{s, t} X=\mathrm{E}_{1}^{s, t}\left\{\operatorname{Tot}_{*} \mathbb{H}_{c} X\right\} \rightarrow \pi_{t-s} \operatorname{Tot}_{\infty} \mathbb{H}_{c} X .
$$

The fiber $F_{s}$ of $\operatorname{Tot}_{s} \mathbb{H}_{c} X \rightarrow \operatorname{Tot}_{s-1} \mathbb{H}_{c} X$ and the homotopy groups of the fiber $F_{s}$ are identified in [BK, Proposition X.6.3] (see also Section 6):

$$
F_{s} \simeq \operatorname{Map}_{*}\left(S^{s}, N X^{s}\right),
$$

where $N X^{s}$ is the fiber of the fibration $\mathbb{H}_{c} X^{s} \rightarrow M^{s-1} \mathbb{H}_{c} X$; see Proposition 3.9. Moreover,

$$
\pi_{i} N X^{s} \cong \pi_{i} \mathbb{H}_{c} X^{s} \cap \operatorname{ker} s^{0} \cap \cdots \cap \operatorname{ker} s^{s-1},
$$

where the maps $s^{i}$ are the cosimplicial degeneracies:

$$
s^{i}: \mathbb{H}_{c} X^{s} \rightarrow \mathbb{H}_{c} X^{s-1} .
$$

Therefore, there is a natural identification

$$
{ }^{\mathbf{T}} \mathrm{E}_{1}^{s, t} X \cong \pi_{t} \mathbb{H}_{c} X^{s} \cap \operatorname{ker} s^{0} \cap \cdots \cap \operatorname{ker} s^{s-1} \quad t \geqslant s \geqslant 0 .
$$

Note that for $t \geqslant 2,{ }^{\mathbf{T}} \mathrm{E}_{1}^{s, t} X$ is the $s$-degree of the normalized cochain complex $N^{*} \pi_{t} \mathbb{H}_{c} X$ associated to $\pi_{t} \mathbb{H}_{c} X$. It is tedious but not hard to check that under this identification, the differential $d_{1}$ of the spectral sequence is chain homotopic to the differential of the normalized cochain complex. Therefore, there are natural identifications

$$
{ }^{\mathbf{T}} \mathrm{E}_{2}^{s, t} X \cong \mathrm{H}^{s}\left(N^{*} \pi_{t} \mathrm{H}_{c}\right) \cong \mathrm{H}^{s}\left(C^{*} \pi_{t} \mathrm{H}_{c} X\right) \cong \mathrm{H}^{s}\left(C^{*} \pi_{t} X\right) \quad t \geqslant 2, t \geqslant s \geqslant 0,
$$

where $C^{*} \pi_{t} X$ denotes the unnormalized cochain complex associated to the cosimplicial abelian group $\pi_{t} X$.

It is not hard to extend these identifications for $t=0$ and $s=0$, and for $t=1$ and $s=0,1$. For a detailed discussion, see [BK, Section X.7].

Define, for a cosimplicial abelian group $A$, the $s$ th cohomotopy group for $s \geqslant 0$ as

$$
\pi^{s} A=\mathrm{H}^{s}\left(C^{*} A\right) .
$$

If $G$ is a cosimplicial pointed set or cosimplicial group, then define $\pi^{0} G$ as the equalizer of $\partial^{0}, \partial^{1}: G^{0} \rightrightarrows G^{1}$. This is a group if $G$ is. 
Similarly, define a pointed cohomotopy set $\pi^{1} G$ for $G$ a cosimplicial group as follows. Let

$$
Z^{1} G=\left\{g \in G^{1}:\left(\partial^{0} g\right)\left(\partial^{1} g\right)^{-1}\left(\partial^{2} g\right)=1\right\} .
$$

There is an action of $G^{0} \times Z^{1} G \rightarrow Z^{1} G$ given by

$$
\left(g_{0}, g_{1}\right) \mapsto\left(\partial^{1} g_{0}\right) g_{1}\left(\partial^{0} g_{0}\right)^{-1} \text {. }
$$

The set $Z^{1} G$ is pointed by the element $1 \in G^{1}$. Let $\pi^{1} G$ be the quotient set of $Z^{1} G$ by this action, pointed by the orbit of $1 \in Z^{1} G$. Then, by [BK, Paragraph X.7.2], there are natural identifications

$$
{ }^{\mathbf{T}} \mathrm{E}_{2}^{s, t} X \cong \begin{cases}\pi^{s} \pi_{t} X & \text { if } t \geqslant s \geqslant 0 \\ 0 & \text { otherwise }\end{cases}
$$

There is another spectral sequence for cosimplicial spaces, which is useful when $X$ is level Kan. This is the homotopy limit spectral sequence, which, in fact, exists in much greater generality. See [BK, Chapter XI]. The main tool is a functor from cosimplicial spaces to cosimplicial spaces called $\Pi$. Let $N \Delta$ be the nerve of the category $\Delta$. Then an element of $N \Delta_{n}$ is a simplex $i_{*}: i_{0} \rightarrow \cdots \rightarrow i_{n}$, where each $i_{k}$ is non-negative integer, and the the arrows are order-preserving maps of the ordered sets associated to $i_{k}:\{0, \cdots, k\}$. For $X$ an arbitrary cosimplicial space, let $\Pi X$ denote the space whose $n$th level is

$$
\Pi^{n} X=\prod_{i_{*} \in N \Delta_{n}} X^{i_{n}}
$$

So, the $n$th level of $\Pi X$ is the product over all compositions $i_{0} \rightarrow i_{n}$ of $X^{i_{n}}$. The face map $\partial^{j}$ for $j<n$ composed with projection onto $i_{*}$ is projection onto $\partial_{j}\left(i_{*}\right)$ followed by the identity. The face map $\partial^{n}$ composed with projection onto $i_{*}$ is projection onto $\partial_{n}\left(i_{*}\right)$ followed by $X\left(i_{n-1} \rightarrow i_{n}\right)$. Similarly, the degeneracy $s^{j}$ followed by projection onto $i_{*}$ is projection onto $s_{j}\left(i_{*}\right)$ followed by the identity.

The important thing about the cosimplicial replacement functor $\Pi$ is that it takes level fibrations into cosimplicial fibrations and preserves weak equivalences. See [BK, Proposition X.5.3].

Definition 4.2. Let $X$ be a cosimplicial pointed space. Let $\operatorname{Ex}^{\infty} X$ denote the cosimplicial pointed space obtained from $X$ by applying the $\mathrm{Ex}^{\infty}$-functor to each level. Then, $\Pi \mathrm{Ex}^{\infty} X$ is fibrant. Define the homotopy limit spectral sequence of $X$, ${ }^{\mathrm{HL}} \mathrm{E}_{1} X$, to be

$$
{ }^{\text {HL }} \mathrm{E}_{1}^{s, t} X=\mathrm{E}_{1}^{s, t}\left\{\operatorname{Tot}_{*} \Pi \mathrm{Ex}^{\infty} X\right\} \rightarrow \pi_{t-s} \operatorname{Tot}_{\infty} \Pi \mathrm{Ex}^{\infty} X .
$$

The space $\operatorname{Tot}_{\infty} \Pi \mathrm{Ex}^{\infty} X$ is called the homotopy limit of $X$, and will be written as holim $\triangle X$.

Lemma 4.3. If $X$ is a cosimplicial pointed space that is level Kan, then the natural morphism $X \rightarrow \mathrm{Ex}^{\infty} X$ induces an isomorphism of spectral sequences

$$
\mathrm{E}_{1}^{s, t}\left\{\boldsymbol{T o t}_{*} \Pi X\right\} \stackrel{\widetilde{\rightrightarrows}}{\rightarrow} \mathrm{E}_{1}^{s, t}\left\{\operatorname{Tot}_{*} \Pi \mathrm{Ex}^{\infty} X\right\} .
$$

This morphism is natural in morphisms of cosimplicial pointed level Kan spaces. 
Let $X$ be an arbitrary pointed cosimplicial space. The functor $\Pi$ can be defined on cosimplicial objects in any category with finite products. In particular, on pointed sets, groups, and abelian groups. There are natural isomorphisms, of cosimplicial pointed sets for $n=0$, cosimplicial groups for $n=1$, and cosimplicial abelian groups for $n>1$,

$$
\pi_{n} \Pi X \simeq \Pi \pi_{n} X
$$

where $\pi_{n} X$ is the cosimplicial object obtained by evaluating $\pi_{n}$ at each cosimplicial level.

For $X$ a cosimplicial object in a category with finite products, there is an natural morphism $X \rightarrow \Pi X$. The maps

$$
X^{n} \rightarrow \prod_{i_{*} \in N \Delta_{n}} X^{i_{n}}
$$

are described as follows. The simplex $i_{*}$ determines a morphism $(0 \rightarrow 1 \rightarrow \cdots \rightarrow n) \rightarrow$ $i_{n}$, by taking the images of 0 from each $i_{i}, 0 \leqslant i<n$. This induces the map $X^{n}$ to the product, and it extends to a cosimplicial map $X \rightarrow \Pi X$.

Proposition 4.4 ([BK, Paragraph XI.7.3]). The canonical map $C^{*} \pi_{n} X \rightarrow C^{*} \Pi \pi_{n} X$ is a quasi-isomorphism.

Proposition 4.5 ([BK, Paragraph XI.7.5]). If $X$ is a fibrant cosimplicial pointed space, then the natural morphism

$$
{ }^{\mathbf{T}} \mathrm{E}_{1} X \rightarrow{ }^{\mathbf{H L}} \mathrm{E}_{1} X
$$

of spectral sequences is an isomorphism.

There is also the Postnikov tower of a cosimplicial space.

Definition 4.6. Let $X$ be a level-fibrant pointed cosimplicial space. Denote by $X(n)$ the level-wise application of the coskeleton functor. Then each $X \rightarrow X(n)$ and $X(m) \rightarrow X(n), m \geqslant n$ is a level fibration. Therefore, $\Pi X(m) \rightarrow \Pi X(n)$ is a fibration for $m \geqslant n$. The spectral sequence of this tower is called the Postnikov spectral sequence for $X$ :

$$
{ }^{\mathbf{P}} \mathrm{E}_{2}^{s, t} X=\tilde{\mathrm{E}}_{2}^{s, t}\left\{\boldsymbol{T o t}_{\infty} \Pi X(*)\right\} \cong \pi_{t-s} \operatorname{Tot}_{\infty} G(t) \rightarrow \pi_{t-s} \operatorname{holim}_{\Delta} X,
$$

where $G(t)$ is the fiber of $\Pi X(t) \rightarrow \Pi X(t-1)$. This fiber is a fibrant resolution of a cosimplicial $K\left(\pi_{t} X, t\right)$-space.

By [BK, Paragraphs XI.7.2-3], there are natural isomorphisms

$$
\pi_{t-s} \operatorname{Tot}_{\infty} G(t) \simeq \pi^{s} \pi_{t} X
$$

for $t \geqslant s \geqslant 0$. Thus,

$$
{ }^{\mathbf{H L}} \mathrm{E}_{2}^{s, t} X \cong{ }^{\mathbf{P}} \mathrm{E}_{2}^{s, t} X
$$

In fact, this isomorphism comes from an isomorphism of spectral sequences. 
Theorem 4.7. Let $X$ be level Kan. Then, there is a natural isomorphism $\phi$ of spectral sequences

$$
\phi:{ }^{\mathrm{HL}} \mathrm{E}_{2} X \rightarrow^{\mathbf{P}} \mathrm{E}_{2} X
$$

from the homotopy limit spectral sequence beginning with the $\mathrm{E}_{2}$-page to the Postnikov tower spectral sequence.

Proof. Recall that to create an isomorphism of spectral sequences that come from exact couples
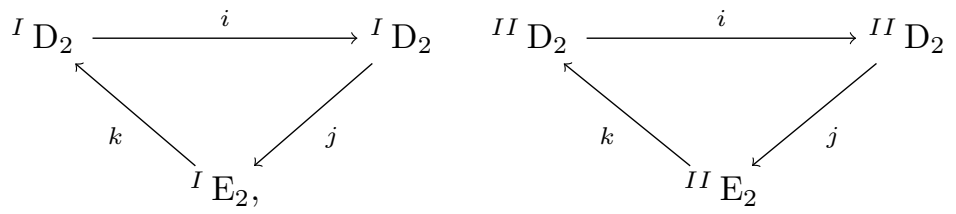

it suffices to create a morphism of exact couples that is an isomorphism just on the E-terms:

$$
\phi:^{I} \mathrm{E}_{2} \stackrel{\simeq}{\rightarrow}{ }^{I} \mathrm{E}_{2} .
$$

Indeed, this is enough to guarantee that the morphism induces an isomorphism on $\mathrm{H}(\mathrm{E})$ and a morphism of the derived couples. So, it follows inductively that this is sufficient. is

Since $X$ is level Kan, there is a double tower of fibrations, of which a typical square

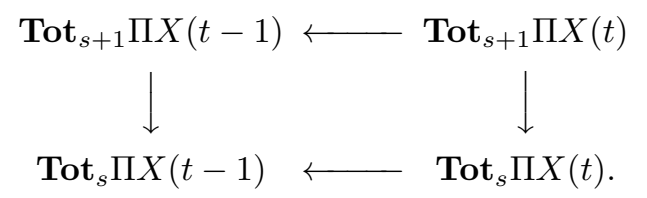

These fit into a bigger diagram

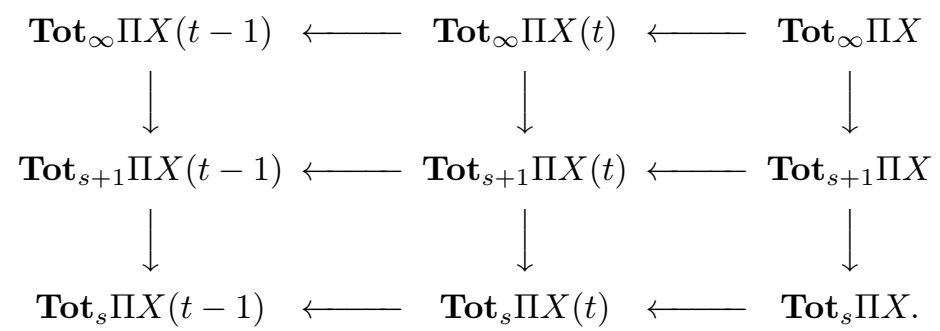

The horizontal inverse limits are $\boldsymbol{T o t}_{s} \Pi X$, and the vertical limits are $\boldsymbol{T o t}_{\infty} \Pi X(t)$. Thus the homotopy limit spectral sequence comes from the tower of fibrations at the horizontal limit, while the Postnikov spectral sequence comes from the tower of fibrations at the vertical limit. Define $F(s+1)$ to be the fiber of $\operatorname{Tot}_{s+1} \Pi X \rightarrow$ $\operatorname{Tot}_{s} \Pi X$, and define $G(t)$ be the fiber of $\boldsymbol{T o t}_{\infty} \Pi X(t) \rightarrow \operatorname{Tot}_{\infty} \Pi X(t-1)$. 
First, construct a morphism

$$
{ }^{\mathbf{H L}} \mathrm{D}_{2}^{s, t}=\operatorname{im}\left(\pi_{t-s} \operatorname{Tot}_{s+1} \Pi X \rightarrow \pi_{t-s} \boldsymbol{T o t}_{s} \Pi X\right) \rightarrow{ }^{\mathbf{P}} \mathrm{D}_{2}^{s, t}=\pi_{t-s} \boldsymbol{T o t}_{\infty} \Pi X(t) .
$$

Let $[x] \in{ }^{\mathbf{H L}} \mathrm{D}_{2}^{s, t}$ be represented by $x: S^{t-s} \rightarrow \boldsymbol{T} t_{s+1} \Pi X \rightarrow \operatorname{Tot}_{s} \Pi X$. By adjunction, view this as

$$
x: \Delta[s]_{+} \wedge S^{t-s} \rightarrow \Delta[s+1]_{+} \wedge S^{t-s} \rightarrow \Pi X .
$$

To create the morphism, one must "lift" this to a morphism $\phi(x): \Delta_{+} \wedge S^{t-s} \rightarrow$ $\Pi X(t)$. The morphism $x$ consists of a compatible collection of morphisms

$$
x^{n, i_{*}}: \Delta^{n}[s]_{+} \wedge S^{t-s} \rightarrow \Delta^{n}[s+1]_{+} \wedge S^{t-s} \rightarrow X^{i_{n}},
$$

one for each $i_{*} \in N \Delta_{n}$. The key point underlying the details below is that $X^{i_{n}}(t)$ is a Kan complex and also has trivial homotopy groups $\pi_{k} X^{i_{n}}(t)$ when $k>t$. At various points one needs to make choices to extend maps. These need to be compatible with the cosimplicial structure. At any given point, this will involve finitely many choices differing in simplicial degrees greater than $t$. Thus, it will always be possible to make the choices compatibly.

Define

$$
\phi(x)^{0, i_{*}}: \Delta^{0}[r]_{+} \wedge S^{t-s} \rightarrow X^{i_{0}}(t)
$$

for all $r$ and $i_{*} \in N \Delta_{0}$ as the composition

$$
\Delta_{+}^{0} \wedge S^{t-s}=\Delta^{0}[s+1]_{+} \wedge S^{t-s} \stackrel{x^{0, i_{*}}}{\longrightarrow} X^{i_{0}} \rightarrow X^{i_{0}}(t) .
$$

Since the coskeleton map is a functor, this definition is functorial.

Now, suppose that

$$
\phi(x)^{h, i_{*}}: \Delta_{+}^{h} \wedge S^{t-s} \rightarrow X^{i_{h}}(t),
$$

is defined for $0 \leqslant h \leqslant k$ and all $i_{*} \in N \Delta_{h}$, compatible with all coface and codegeneracy maps in this range. In other words, suppose that we have defined compatible maps

$$
\phi(x)^{h, *}: \Delta_{+}^{h} \wedge S^{t-s} \rightarrow \Pi^{h} X(t)
$$

for $0 \leqslant h \leqslant k$. If $i_{*} \in N \Delta_{k+1}$ is degenerate, then define

$$
\phi(x)^{k+1, i_{*}}: \Delta_{+}^{k+1} \wedge S^{t-s} \rightarrow X^{i_{k+1}}(t)
$$

by forcing the diagram

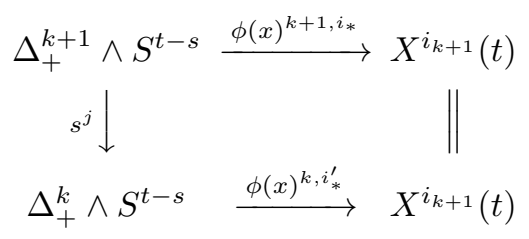

to be commutative for every $j$ such that $s_{j}\left(i_{*}^{\prime}\right)=i_{*}$ for some $i_{*}^{\prime} \in N \Delta_{k}$. Making these simultaneously commutative for all choices of $j$ is possible because of the simplicial relations $s_{i} \circ s_{j}=s_{j+1} \circ s_{i}$ for $i \leqslant j$. 
If $i_{*} \in N \Delta_{k+1}$ is not degenerate, then the cosimplicial structure requires that the diagrams

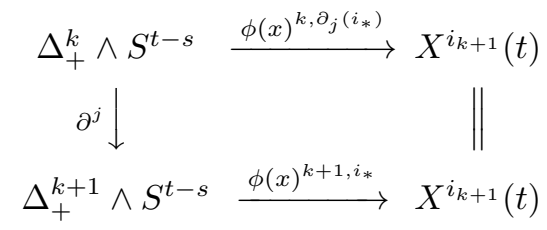

for $0 \leqslant j<k$ and

$$
\begin{array}{cc}
\Delta_{+}^{k} & \wedge S^{t-s} \stackrel{\phi(x)^{k, \partial_{k}\left(i_{*}\right)}}{\longrightarrow} X^{i_{k}}(t) \\
\partial^{k} \downarrow & X\left(i_{k+1} \rightarrow i_{k}\right) \downarrow \\
\Delta_{+}^{k+1} \wedge S^{t-s} & \stackrel{\phi(x)^{k+1, i_{*}}}{\longrightarrow} X^{i_{k+1}}(t)
\end{array}
$$

be commutative. In other words, the map $\phi(x)^{k+1, i_{*}}$ is determined on $\partial \Delta_{+}^{k+1} \wedge S^{t-s}$.

Thus, to make the inductive step, one must fill in the dashed line so that the diagram

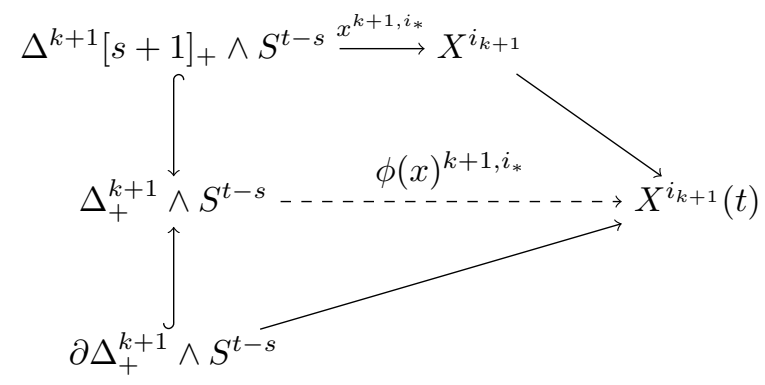

is commutative. If $s \geqslant k$, then $\Delta^{k+1}[s+1]_{+}=\Delta_{+}^{k+1}$, so there is nothing to do. If $s<k$, then $\Delta^{k+1}[s+1]_{+} \wedge S^{t-s} \subseteq \partial \Delta_{+}^{k+1} \wedge S^{t-s}$, and the outer square commutes by induction. Therefore, it suffices for the dashed arrow to commute in the bottom triangle. As $s<k$, the arrow $\partial \Delta_{+}^{k+1} \wedge S^{t-s} \rightarrow X^{i_{k+1}}$ corresponds to a map $S^{k+t-s+1} \rightarrow X^{i_{k+1}}(t)$. But, $k+t-s+1>t$. Hence, $\pi_{k+t-s+1} X^{i_{k+1}}(t)=0$. As $X(t)$ is a Kan complex, such a fill exists (see [GJ, page 35]).

Choose a fill for all choices of $i_{*}$. This completes the induction, giving

$$
\phi(x)^{*}: \Delta_{+}^{*} \wedge S^{t-s} \rightarrow \Pi^{*} X(t),
$$

for $0 \leqslant * \leqslant k+1$. The process outlined earlier in the proof gives a base case. So, induction provides the desired map

$$
\phi(x): \Delta_{+} \wedge S^{t-s} \rightarrow \Pi^{*} X(t) .
$$

If $y$ is another morphism $S^{t-s} \rightarrow \operatorname{Tot}_{s+1} \Pi X$ representing the class $[x]$, then it is straightforward, using a similar inductive argument, to lift a homotopy between $x$ and $y$ to a homotopy between $\phi(x)$ and $\phi(y)$ so that the map is well-defined on the level of homotopy groups. Thus, the construction above determines a well-defined the $\operatorname{map} \phi:{ }^{\mathbf{H L}} \mathrm{D}_{2}^{s, t} \rightarrow{ }^{\mathbf{P}} \mathrm{D}_{2}^{s, t}$. 
It is easy to see that $\phi$ commutes with $i$. Indeed, on ${ }^{\mathbf{H L}} \mathrm{D}_{2}, i$ is given by restriction from $\Delta[s]$ to $\Delta[s-1]$. On ${ }^{\mathbf{P}} \mathrm{D}_{2}, i$ is given by mapping $X(t) \rightarrow X(t-1)$. Now, composing $\phi(x)$ with $\Pi X(t)$ gives a map that solves the lifting problem to define $\phi(i(x))$ :

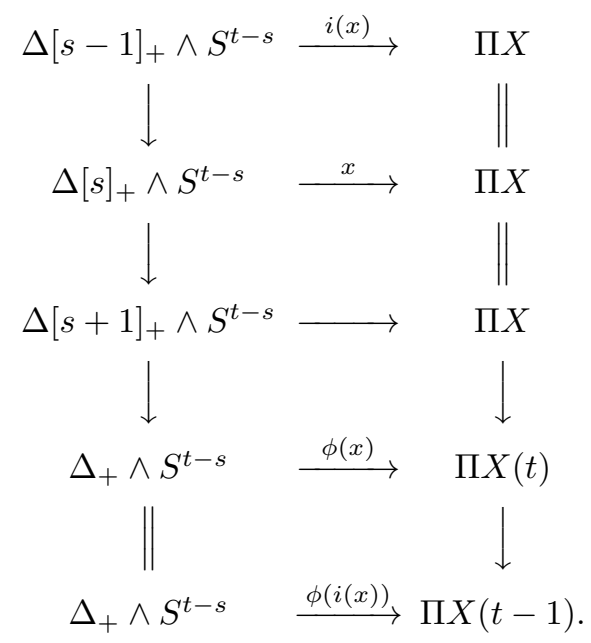

Therefore, $\phi$ commutes with $i$.

Extend $\phi$ to ${ }^{\mathrm{HL}} \mathrm{E}_{2}$ by lifting

$$
x: S^{t-s} \rightarrow F(s)
$$

to

$$
x: S^{t-s} \rightarrow \operatorname{Tot}_{s+1} \Pi X
$$

and applying $\phi$ (recall the description in Section 2 of $\mathrm{E}_{2}$ ). In other words, apply $k$ and then $\phi$. This defines an element of $\mathbf{P}^{s} \mathrm{D}_{2}^{s, t}$, and, by construction, $\phi(x)$ actually factors through the fiber $G(t)$. Indeed, since

$$
x: \Delta[s+1] \wedge S^{t-s} \rightarrow \Pi X
$$

comes from the fiber $F_{s}$, it restricts to the trivial map on

$$
\Delta[s-1] \wedge S^{t-s} .
$$

Therefore, $x$ is trivial on the $t-1$-skeleton. Extending this to a map

$$
x: \Delta \wedge S^{t-s} \rightarrow \Pi X(t)
$$

as above does not change this, so that when one composes with $\Pi X(t) \rightarrow \Pi X(t-1)$, the map is homotopic to the constant map on the basepoint. To check that this determines a well-defined map on $\mathrm{E}_{2}$-terms for $t-s>0$, one must check that if

$$
x=y+j(z),
$$

where $z \in \operatorname{ker}\left(\pi_{t-s+1} X_{s-1} \rightarrow \pi_{t-s+1} X_{s-2}\right)$ and

$$
x, y \in \operatorname{ker}\left(\pi_{t-s} F(s) \rightarrow \pi_{t-s} X_{s} / i\left(\pi_{t-s} X_{s+1}\right)\right),
$$

then $\phi(x)=\phi(z)$. The equation $x=y+j(z)$ makes sense for $t-s>1$ and for $t-s=$ 1 , since $\pi_{2} X_{s-1} \rightarrow \pi_{1} F_{s}$ lands in the center of $\pi_{1} F_{s}$ (recall Construction 2.1). But, 
since

$$
\phi(j(z))=\phi(k(j(z)))=\phi(0),
$$

it follows that this definition of $\phi$ on ${ }^{\mathbf{H L}} \mathrm{E}_{2}$, for $t-s>0$ is indeed well-defined. When $t-s=0$, it is necessary to show that if $x, y \in \operatorname{ker}\left(\pi_{0} F(s) \rightarrow \pi_{0} X_{s} / i\left(\pi_{0} X_{s+1}\right)\right)$ and $z \in \operatorname{ker}\left(\pi_{1} X_{s-1} \rightarrow \pi_{1} X_{s-2}\right)$ are such that $x=z \cdot y$, then $\phi(x)=\phi(y)$. However, the equation $x=z \cdot y$ implies that $k(x)=k(y)$, since $\pi_{0} F(s) \rightarrow \pi_{0} X_{s}$ factors through the quotient onto the set of orbits of $\pi_{0} F(s)$ under the action of $\pi_{1} X_{s-1}$ (Construction 2.1). Thus, $\phi(x)=\phi(k(x))=\phi(k(y))=\phi(y)$.

It remains only to check that $\phi$ respects $j$. Again, let $[x] \in \pi_{t-s} \boldsymbol{T}_{s} \Pi X$ be represented by

$$
x: \Delta[s+1]_{+} \wedge S^{t-s} \rightarrow \Pi X .
$$

Recall that $j([x])$ is obtained by the map

$$
\pi_{t-s} \operatorname{Tot}_{s+1} \Pi X \rightarrow \pi_{t-s-1} F(s+2),
$$

given by lifting a horn

$$
\Delta[s+1]_{+} \wedge \Lambda_{i,+}^{s-t} \rightarrow \Delta[s+1]_{+} \wedge S^{s-t} \rightarrow \Pi X
$$

to

$$
\Delta[s+2]_{+} \wedge \Delta_{+}^{s-t} \rightarrow \Pi X
$$

and then restricting to the $i$ th face to obtain

$$
\Delta[s+2]_{+} \wedge S^{t-s-1} \rightarrow \Pi X,
$$

which by adjunction is in $\pi_{t-s-1} F(s+2)$. Lift

$$
\Delta[s+2]_{+} \wedge \Delta_{+}^{s-t} \rightarrow \Pi X
$$

as above to a map

$$
\Delta_{+} \wedge \Delta_{+}^{s-t} \rightarrow \Pi X(t+1) .
$$

By construction, it maps down to

$$
\phi(x): \Delta_{+} \wedge S^{t-s} \rightarrow \Pi X(t),
$$

while the restriction to $\Delta_{+} \wedge S^{t-s-1}$ is in the fiber $G(t+1)$. Therefore, $\phi$ commutes with $j$.

To prove that $\phi$ is injective on $\mathrm{E}_{2}$, let $x \in{ }^{\mathbf{H L}} \mathrm{E}_{2}^{s, t}$ be represented by

$$
x: \Delta[s+1]_{+} \wedge S^{t-s} \rightarrow \Pi X,
$$

and let

$$
\phi(x): \Delta_{+} \wedge S^{t-s} \rightarrow \Pi X(t)
$$

factor through $G(t)$. Suppose that $\phi(x)$ is homotopic to the constant map in $G(t)$, and let

$$
y: \Delta_{+}^{1} \wedge \Delta_{+} \wedge S^{t-s} \rightarrow G(t) \rightarrow \Pi X(t)
$$

be such a homotopy. It is possible to find an extension 


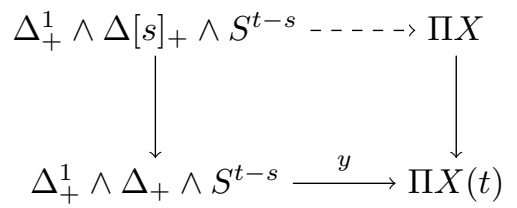

giving a homotopy between $x$ and the constant map by definition of the coskeleton $\Pi X(t)$. Therefore, $\phi$ is injective on $\mathrm{E}_{2}$.

To show that $\phi$ is surjective on $\mathrm{E}_{2}$, let

$$
y: S^{t-s} \rightarrow G(t)
$$

be represented by

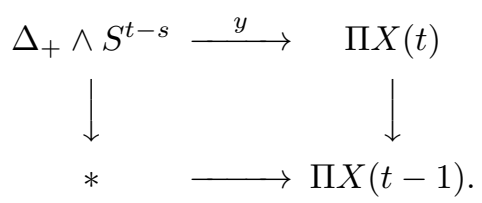

A lift $x$ for the diagram

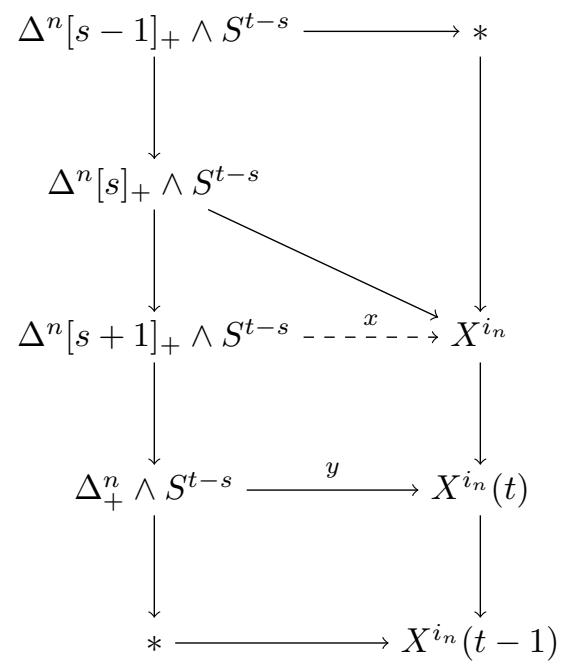

exists by definition for $n \leqslant s$. For $n>s$, the top quadrilateral means that the map

$$
\Delta^{n}[s]_{+} \wedge S^{t-s} \rightarrow X^{i_{n}}
$$

is a diagram of $t-s$-spheres in $\Omega^{s} X^{i_{n}}$, and finding a lift $x$ is the same as saying that this class is 0 in $\pi_{t-s} \Omega^{s} X^{i_{n}}$. But, the map $y$ in the diagram says that this class is 0 in $\pi_{t-s} \Omega^{s} X^{i_{n}}(t)$. Once again, by definition of the coskeleton functor, it follows that it is already 0 in $\pi_{t-s} \Omega^{s} X^{i_{n}}$. Now, arguing as above, one may inductively choose extensions in the diagram to create an element $x$ of ${ }^{H L} \mathrm{E}_{2}^{s, t}$ such that $\phi(x)=y$. Therefore, $\phi$ is also surjective on $\mathrm{E}_{2}$.

Remark 4.8. This theorem does not appear to be new. Indeed, it appears that Thomason was aware of it: see [T, page 542, Paragraph 3]. However, we know of no reference 
for a proof. It in fact holds in the greater generality of presheaves of simplicial sets on small categories.

Corollary 4.9. Suppose that $X$ is a level Kan cosimplicial pointed space. If one of the spectral sequences

$$
\begin{gathered}
{ }^{\text {HL }} \mathrm{E}_{1} X \rightarrow \pi_{t-s} \underset{\Delta}{\operatorname{holim}} X \\
{ }^{\mathbf{P}} \mathrm{E}_{2} X \rightarrow \pi_{t-s} \operatorname{holim} X .
\end{gathered}
$$

converges, then both do, and the filtrations ${ }^{\mathbf{H L}} Q_{s}$ and ${ }^{\mathbf{P}} Q_{s}$ coincide on $\pi_{*}$ holim $\Delta$.

\section{The Čech approximation}

Let $C$ be a Grothendieck site with terminal object $U$. Denote by $\operatorname{Pre}(C)$ the category of presheaves on $C$, and write $\mathbf{s P r e}(C)$ for the category of simplicial presheaves.

We use the following closed model category structure on simplicial presheaves, called the local model category structure. The cofibrations are the pointwise cofibrations. Thus, $X \rightarrow Y$ is a cofibration if and only if $X(V) \rightarrow Y(V)$ is a monomorphism for every object $V$ of $C$. For an object $V$ of $C$, there is a site with terminal object $C_{/ V}$. Each presheaf on $C$ restricts to a presheaf on $C_{/ V}$. For a simplicial presheaf $X$, an object $V$ of $C$, and a basepoint $x \in X(V)_{0}$, there are presheaves of homotopy groups $\pi_{k}^{p}(X \mid V, x)$ :

$$
(f: W \rightarrow V) \mapsto \pi_{k}\left(|X(W)|, f^{*}(x)\right)
$$

where $|X(W)|$ denotes the geometric realization of the simplicial set $X(W)$. Let $\pi_{k}(X \mid V, x)$ be the associated homotopy sheaf. Call $w: X \rightarrow Y$ a weak equivalence if it induces an isomorphism of homotopy sheaves

$$
\pi_{k}(X \mid V, x) \stackrel{\simeq}{\rightarrow} \pi_{k}(Y \mid V, w(x))
$$

for all choices of $V$, all basepoints $x$ of $X(V)$, and all $k \geqslant 0$. The fibrations are all maps having the right lifting property with respect to all cofibrations that are simultaneously weak equivalences (the acyclic cofibrations). That this is a simplicial closed model category is due to Joyal; for a proof, see Jardine's paper [J]. Refer to these classes of morphisms more specifically as global fibrations, global cofibrations, and local weak equivalences.

Theorem 5.1 (Dugger-Hollander-Isaksen [DHI]). If $X$ is a simplicial presheaf, and if $\mathcal{V}^{\bullet} \rightarrow V$ is a hypercover in $C$, then let $X_{\mathcal{V}}$ denote the cosimplicial space associated to $\mathcal{V}^{\bullet}$. There is a canonical augmentation $X(V) \rightarrow X_{\mathcal{V}}$. The simplicial presheaf $X$ is globally fibrant if and only if

$$
X(V) \rightarrow \underset{\Delta}{\operatorname{holim}} X_{\mathcal{V}} \bullet
$$

is a weak equivalence for all hypercovers $\mathcal{V}^{\bullet} \rightarrow V$ in $C$.

There are other types of morphisms, namely pointwise weak equivalences and pointwise fibrations. A pointwise weak equivalence is a morphism $f: X \rightarrow Y$ such that $X(V) \stackrel{\sim}{\rightarrow} Y(V)$ is a weak equivalence of simplicial sets for all objects $V$ of $C$. Two pointwise weak equivalent sheaves are local weak equivalent, and two local weak 
equivalent fibrant presheaves are pointwise weak equivalent. A pointwise fibration is a morphism $f: X \rightarrow Y$ such that every $f: X(V) \rightarrow Y(V)$ is a fibration of simplicial sets. Say that $X$ is pointwise Kan or pointwise fibrant if $X \rightarrow *$ is a pointwise fibration

Note that if a simplicial presheaf is pointed, then the homotopy presheaves and sheaves above may be defined globally.

Let $F$ be a functor from simplicial sets to simplicial sets such that $F(\emptyset)=\emptyset$, or from pointed simplicial sets to pointed simplicial sets such that $F(*)=*$. If $X$ is a simplicial presheaf, denote by $F X$ the pointwise application of $F$ to $X$, so that $(F X)(V)=F(X(V))$ for all $V$. For instance, below, $\operatorname{cosk}_{n} X$ will be the pointwise $n$ coskeleton of $X$. If $F$ preserves weak equivalences of simplicial sets, then it preserves local weak equivalences of simplicial presheaves. This is the case, for instance, for the coskeleta functors and for the Ex functor. In particular, one may always replace $X$ with the pointwise weakly equivalent presheaf $\operatorname{Ex}^{\infty} X$, which is pointwise Kan.

Definition 5.2. If $X$ is a presheaf of pointed spaces, and if $X \rightarrow \mathbb{H} X$ is a pointed fibrant resolution of $X$, then $\mathrm{H} X$ is called the hypercohomology space of $X$. Hypercohomology sets, groups, and abelian groups are defined by

$$
\mathrm{H}^{s}(U, X)=\pi_{s} \Gamma(U, \mathrm{H} X) .
$$

Definition 5.3. Let $X$ be a presheaf of pointed simplicial sets on $C$, and let $X \rightarrow \mathbb{H} X$ be a pointed fibrant resolution. Finally, let $X(n)$ be a pointed fibrant resolution of $\operatorname{cosk}_{n} \mathrm{H} X$ so that $X(n) \rightarrow X(n-1)$ is a global fibration for all $n \geqslant 0$. Then, the Brown-Gersten spectral sequence associated to $X$ is the $\tilde{\mathrm{E}}_{2}$-spectral sequence (see Section 2) associated to the tower of fibrations

$$
\Gamma(U, X(n)) \rightarrow \Gamma(U, X(n-1))
$$

That is, define the Brown-Gersten spectral sequence as

$$
{ }^{\mathbf{B G}} \mathrm{E}_{2}^{s, t} X=\tilde{\mathrm{E}}_{2}^{s, t}\{\Gamma(U, X(*))\} \rightarrow \pi_{t-s} \lim _{\leftarrow} \Gamma(U, X(n)) .
$$

This is also called the descent spectral sequence.

Lemma 5.4 ([GS]). Suppose that the site $C$ is locally of finite cohomological dimension. Then, the natural morphism

$$
\Gamma(U, \mathbb{H} X) \rightarrow \lim _{\leftarrow} \Gamma(U, X(n))
$$

is a weak equivalence for all objects $U$ whenever $X$ is locally weak equivalent to a product of a constant pointed space and a connected space.

In the situation of the lemma, write the Brown-Gersten spectral sequence as

$$
\text { BG } \mathrm{E}_{2} X \rightarrow \mathrm{H}^{t-s}(U, X) \text {. }
$$

For instance, the lemma holds for presheaves of Quillen $K$-theory spaces on the étale sites of schemes of finite étale cohomological dimension.

The theorem of Dugger-Hollander-Isaksen, Theorem 5.1, says that the natural map

$$
\Gamma(U, X(n)) \rightarrow \operatorname{Tot}_{\infty} \Pi X(n)_{\mathcal{U}} \bullet
$$

is an isomorphism whenever $\mathcal{U}^{\bullet} \rightarrow U$ is a hypercover, and $X(n)_{\mathcal{U}}$. denotes the cosimplicial space obtained by evaluating $X(n)$ at each level of $\mathcal{U}^{\bullet}$. 
Definition 5.5. Let $\mathcal{U}^{\bullet}$ be a hypercover of an object $U$ of $C$. Let $X$ be a simplicial presheaf. Then, let $X_{\mathcal{U}}$ • denote the cosimplicial space one gets by evaluating $X$ at $\mathcal{U}^{\bullet}$. The Cech hypercohomology of $X$ on $\mathcal{U}^{\bullet}$ is defined as

$$
\check{\mathrm{H}}^{s}\left(\mathcal{U}^{\bullet}, X\right)=\pi_{s} \operatorname{Tot}_{\infty} \mathbb{H}_{c} X_{\mathcal{U}} .
$$

Definition 5.6. The Čech hypercohomology spectral sequence associated to $X$ and $\mathcal{U}^{\bullet} \rightarrow U$ is the total space spectral sequence associated to $\mathbb{H}_{c} X_{\mathcal{U}} \bullet$ :

$$
\mathcal{U}^{\bullet} \mathrm{E}_{1} X={ }^{\mathbf{T}} \mathrm{E}_{1} X_{\mathcal{U}} \bullet
$$

By Equation (8), the $\mathrm{E}_{2}$-terms for the corresponding spectral sequence are naturally identified with

$$
\mathrm{E}_{2}^{s, t} \simeq \check{\mathrm{H}}^{s}\left(\mathcal{U}^{\bullet}, \pi_{t}^{p} X\right)
$$

as desired.

There is a natural morphism from Čech hypercohomology to hypercohomology induced by a natural morphism

$$
\operatorname{Tot}_{\infty} \mathbb{H}_{c} X_{\mathcal{U}} \bullet \Gamma(U, \mathbb{H} X)
$$

This morphism is the composition of

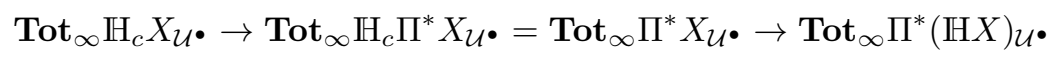

with the inverse of the local weak equivalence (Theorem 5.1)

$$
\Gamma(U, \mathbb{H} X) \stackrel{\sim}{\rightarrow} \operatorname{Tot}_{\infty} \Pi^{*}(\mathbb{H} X)_{\mathcal{U}} \bullet
$$

Theorem 5.7. Let $X$ be a pointed simplicial presheaf, and let $\mathcal{U}^{\bullet} \rightarrow U$ be a hypercover. There is a natural morphism

$$
\mathcal{u}^{\bullet} \mathrm{E}_{2} X \rightarrow{ }^{\mathrm{BG}} \mathrm{E}_{2} X
$$

from the Čech hypercohomology spectral sequence to the Brown-Gersten spectral sequence, which on $\mathrm{E}_{2}$-terms is the natural map

$$
\check{\mathrm{H}}^{s}\left(\mathcal{U}^{\bullet}, \pi_{t}^{p} X\right) \rightarrow \mathrm{H}^{s}\left(U, \pi_{t} X\right),
$$

and which respects the morphism on abutments of Equation (11).

Proof. One may assume, possibly by applying the $\mathrm{Ex}^{\infty}$ that $X$ is pointwise Kan. Therefore, $X_{\mathcal{U}} \bullet$ is a level Kan cosimplicial pointed space. Then, the space $\Pi X_{\mathcal{U}} \bullet$ is fibrant, so that there is a natural morphism

$$
\mathbb{H}_{c} X_{\mathcal{U}} \bullet \rightarrow \Pi X_{\mathcal{U}} \bullet
$$

making the diagram

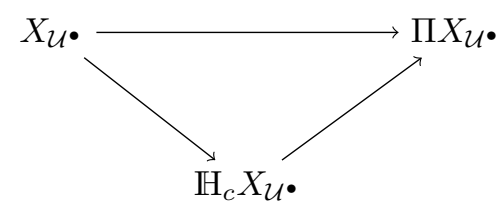


commutative. This induces a morphism of total space spectral sequences

$$
\mathcal{U}^{\bullet} \mathrm{E}_{2} X \rightarrow{ }^{\mathbf{T}} \mathrm{E}_{2} \mathrm{H}_{c} X_{\mathcal{U}} \bullet \rightarrow{ }^{\mathbf{H L}} \mathrm{E}_{2} X_{\mathcal{U}} \bullet
$$

There is a morphism

$$
{ }^{\mathbf{H L}} \mathrm{E}_{2} X_{\mathcal{U}} \bullet \rightarrow{ }^{\mathbf{H L}} \mathrm{E}_{2}(\mathrm{H} X)_{\mathcal{U}} \bullet
$$

induced by $X \rightarrow \mathbb{H} X$. Theorem 4.7 furnishes an isomorphism

$$
{ }^{\mathrm{HL}} \mathrm{E}_{2}(\mathrm{H} X)_{\mathcal{U}} \bullet \stackrel{\simeq}{\rightarrow} \mathrm{P} \mathrm{E}_{2}(\mathrm{H} X)_{\mathcal{U}} \bullet
$$

Again, the fibrant replacement $\operatorname{cosk}_{n} \mathbb{H} X \rightarrow X(n)$ induces a morphism

$$
{ }^{\mathbf{P}} \mathrm{E}_{2}(\mathrm{H} X)_{\mathcal{U}} \bullet \rightarrow \tilde{\mathrm{E}}_{2}^{s, t}\left\{\boldsymbol{T o t}_{\infty} \Pi X(n)_{\mathcal{U}} \bullet\right\} .
$$

Finally, the result of Dugger-Hollander-Isaksen [DHI] says that the natural morphism

$$
{ }^{\mathrm{BG}} \mathrm{E}_{2} X \rightarrow \tilde{\mathrm{E}}_{2}^{s, t}\left\{\operatorname{Tot}_{\infty} \Pi X(n)_{\mathcal{U}} \bullet\right.
$$

is in fact an isomorphism.

The theorem follows by taking the composition of the morphisms of Equations (12), (13), (14), and (15), and the inverse of the morphism of Equation (16).

\section{Differentials in the holim spectral sequence}

Lemma 6.1 ([BK, Proposition X.6.3.i]). Take $X$ to be a fibrant pointed cosimplicial space. There are equivalences $F(s) \simeq \operatorname{Map}_{*}\left(S^{s}, N X^{s}\right)$, where $F(s)$ is the fiber of $\operatorname{Tot}_{s} X \rightarrow \operatorname{Tot}_{s-1} X$, and $N X^{s}$ is the fiber of the fibration $X^{s} \rightarrow M^{s-1} X$.

Sketch of proof. Let $\beta: \Delta^{n} \rightarrow F(s)$. By adjunction, this is a morphism

$$
\Delta[s]_{+} \wedge \Delta_{+}^{n} \stackrel{\beta}{\rightarrow} X
$$

such that the restriction to $\Delta[s-1]_{+} \wedge \Delta_{+}^{n}$ factors through the base-point. In particular, the level $s$ picture is a map of pointed spaces $\Delta_{+}^{s} \wedge \Delta_{+}^{n} \rightarrow X^{s}$ such that the restriction to the $s-1$-skeleton on the left $\Delta^{s}[s-1]_{+} \wedge \Delta_{+}^{n}$ factors through the base-point of $X^{s}$. Therefore, this is a morphism $S^{s} \wedge \Delta_{+}^{n} \rightarrow X$. That is, $\beta$ determines an $n$-simplex of $\operatorname{Map}_{*}\left(S^{s}, X^{s}\right)$. However, the degeneracy $s^{i} \beta: \Delta^{s-1}[s]_{+} \wedge \Delta_{+}^{n} \rightarrow$ $X^{s-1}$ factors through $\Delta^{s-1}[s-1]_{+} \wedge \Delta_{+}^{n}$ and so is trivial. Therefore the $n$-simplex of $\operatorname{Map}_{*}\left(S^{s}, X^{s}\right)$ actually lives in $\operatorname{Map}_{*}\left(S^{s}, N X^{s}\right)$. Conversely, suppose that $\gamma$ is an $n$-simplex of $\operatorname{Map}_{*}\left(S^{s}, N X^{s}\right)$. Again, by adjunction, view this as a map $S^{s} \wedge \Delta_{+}^{n} \rightarrow$ $N X^{s} \rightarrow X^{s}$. Extend this as follows into a map $\Delta[s]_{+} \wedge \Delta_{+}^{n} \rightarrow X$. The lift of $\gamma$ to $\Delta_{+}^{s} \wedge \Delta_{+}^{n}$ is the $s$-level of this morphism. Since $\lambda$ factors through $*$ for any degeneracy on $\Delta^{s}$, let $\Delta^{k}[s]_{+} \wedge \Delta_{+}^{n} \rightarrow * \rightarrow X^{k}$ for $k<s$. If $k>s$, use the following diagram:

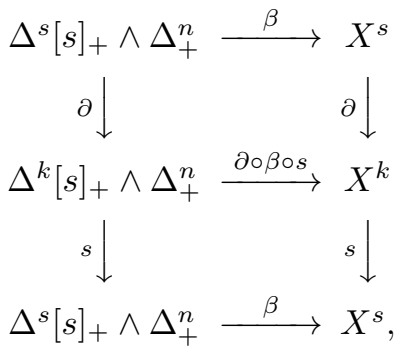


where the face map $\partial$ (resp. the degeneracy map $s$ ) is some composition of face (resp. degeneracy) maps such that the vertical compositions are the identity. This defines the extension inductively on the faces of $\Delta^{k}[s]$. But, one need only define it up to faces, since $k>s$. This gives us an $n$-simplex of $\boldsymbol{T} \boldsymbol{t}_{s} X$. Clearly, these constructions are mutually inverse.

It is not hard, using this identification, to show that the homotopy groups of $F(s)$ are the groups of the normalized cochain complexes associated to $X$. That is

$$
\pi_{t-s} F(s) \simeq \pi_{t-s} \operatorname{Map}_{*}\left(S^{s}, N X^{s}\right) \simeq \pi_{t} X^{s} \cap \operatorname{ker} s^{0} \cap \cdots \cap \operatorname{ker} s^{s-1},
$$

where $\operatorname{ker} s^{i}$ is $\operatorname{ker}\left(s^{i}: \pi_{t} X^{s} \rightarrow \pi_{t} X^{s-1}\right)$. See [BK, Proposition X.6.3].

The differentials in the homotopy limit spectral sequence are those of the spectral sequence associated to a tower of fibrations. In particular, they arise from trying to lift simplices to higher and higher levels of the tower. The recipe is as follows: let

$$
\cdots \rightarrow X_{s+1} \rightarrow X_{s} \rightarrow X_{s-1} \rightarrow \cdots
$$

be a tower of fibrations, and let $\delta: S^{t} \rightarrow X_{s-1}$ be a homotopy class. Writing $S^{t}$ as the quotient of $\Delta^{t}$ by its boundary, we get a map $\Delta^{t} \rightarrow X_{s-1}$ that is trivial on the boundary $\partial \Delta^{t}$. We can obviously then map any horn $\Lambda_{i}^{t}$ to $X^{s}$ by sending it to the base-point. Then, there is a lifting problem

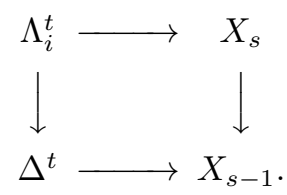

Since the map on the right is a fibration, we do get a lift to a map $\Delta^{t} \rightarrow X_{s}$. However, it may no longer map the boundary of $\Delta^{t}$ to the base-point of $X_{s}$. Instead, the $i$ th face determines a map $\Delta^{t-1} \rightarrow X_{s}$ that $i s$ trivial on the boundary. The corresponding class in $\pi_{n-1} F(s)$ is the obstruction to lifting the homotopy class of $\delta$ to a homotopy class on $X_{s}$.

Below, the tower of fibrations is the total space tower associated to a fibrant cosimplicial space (see Section 3).

Remark 6.2. We briefly indicate how to show that $d_{1}$ is homotopic to the cochain differential on the normalized cochain complex for $\pi_{t} X$. Let $\beta$ this time represent a class in $\pi_{t-s} \operatorname{Map}_{*}\left(S^{s}, N X^{s}\right)$. This determines a corresponding map $S^{s} \wedge S^{t-s} \rightarrow X^{s}$. Extending this, one gets a map $\Delta[s]_{+} \wedge S^{t-s} \rightarrow X$. Lift this, using some horn $\Lambda_{i}^{t-s}$, to a map $\Delta[s+1]_{+} \wedge \Delta_{+}^{t-s} \rightarrow X$. On $\Delta[s]_{+} \wedge \Delta_{+}^{t-s}$ this agrees with $\beta$. Restricting to the $i$ th face of $\Delta^{t-s}$, one gets a map $\Delta[s+1]_{+} \wedge S^{t-s-1} \rightarrow X$. Then, look at just $\Delta_{+}^{s+1} \wedge S^{t-s-1} \rightarrow X^{s+1}$, and check that one lands in the fiber. The reason that this agrees with the alternating sum $d=\Sigma(-1)^{i} d^{i}$ is that the simplices $\Delta_{+}^{s+1} \wedge \Delta_{+}^{t-s} \rightarrow$ $X^{s+1}$ give a homotopy between $d(\beta)$ and $d_{1}(\beta)$.

Construction 6.3. Let $\delta: S^{t} \rightarrow X^{s}$ represent a class $[\delta]$ of $\pi^{s} \pi_{t} X$, where $t-s \geqslant 0$, and suppose that $\delta$ factors as $\delta: S^{t-s} \rightarrow \operatorname{Map}_{*}\left(S^{s}, N X^{s}\right)$. That is, suppose that $\delta$ represents $[\delta]$ in the normalized chain complex. Then, as in the proof of Lemma 6.1, 
extend $\delta$ to a map

$$
\delta^{\prime}: \Delta[s]_{+} \wedge \Delta_{+}^{t-s} \rightarrow X .
$$

Now, the restriction of this map to $\Delta[s]_{+} \wedge \partial \Delta_{+}^{t-s}$ factors through the base-point. Choosing a horn $\Lambda_{i}^{t-s} \subset \partial \Delta^{t-s}$ and the map $\Delta[s+1]_{+} \wedge\left(\Lambda_{i}^{t-s}\right) \rightarrow * \rightarrow X$, one gets, using the fact that $\operatorname{Tot}_{s+1} X \rightarrow \operatorname{Tot}_{s} X$ is a fibration, a lift to a map

$$
\gamma: \Delta[s+1]_{+} \wedge \Delta_{+}^{t-s} \rightarrow X
$$

such that the following diagram commutes

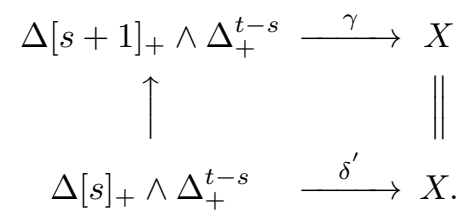

The fact that $\delta$ represents a homotopy class implies, by Remark 6.2 , that the restriction of $\gamma$ to

$$
\Delta_{+}^{s+1} \wedge \partial_{i} \Delta_{+}^{t-s} \rightarrow X^{s+1}
$$

is contractible in $\operatorname{Map}_{*}\left(S^{s+1}, N X^{s+1}\right)$. Therefore, one can replace $\gamma$ by a homotopic map $\gamma^{\prime}$ such that the restriction to $\gamma^{\prime}: \Delta[s+1]_{+} \wedge \partial \Delta_{+}^{t-s} \rightarrow X$ factors through the base-point. Thus, one can repeat the process, using a trivial map

$$
\Delta[s+2]_{+} \wedge\left(\Lambda_{j}^{t-s}\right)_{+} \rightarrow * \rightarrow X,
$$

to get a lift $\epsilon: \Delta[s+2]_{+} \wedge \Delta_{+}^{t-s} \rightarrow X$, using some horn $\Lambda_{j}^{t-s}$. The differential $d_{2}([\delta])$ is the class of $\epsilon: \Delta_{+}^{s+2} \wedge \partial_{j} \Delta_{+}^{t-s} \rightarrow X^{s+2}$ in $\pi^{s+2} \pi_{t-s-1} X$.

Note the following, which will be an aid to making the extensions described above. We claim that to extend $\Delta[s]_{+} \wedge \Delta_{+}^{t-s} \rightarrow X$ to $\Delta[s+1]_{+} \wedge \Delta_{+}^{t-s}$ it is sufficient to describe the extension of $\Delta^{s+1}[s]_{+} \wedge \Delta_{+}^{t-s} \rightarrow X^{s+1}$ to $\Delta_{+}^{s+1} \wedge \Delta_{+}^{t-s}$. Indeed, make the same argument as in the proof of Lemma 6.1, especially the argument using Diagram (17).

\section{Description of $d_{2}$ for the Čech spectral sequence}

The descriptions of the differentials above translate into the following theorem in the setting of the Čech approximation.

Theorem 7.1. Let $X$ be a presheaf of pointed simplicial sets on the site $C$, and let $\mathcal{U}^{\bullet}: \mathcal{V}_{A} \rightarrow \mathcal{U}_{I} \rightarrow U$ be a 1-hypercover in C. Thus, for $\alpha \in A, V_{i j}^{\alpha} \rightarrow U_{i j}$ is a covering morphism, where $U_{i j}=U_{i} \times_{U} U_{j}$. Then, for $t>0$, the differentials

$$
d_{2}: \check{\mathrm{H}}^{0}\left(\mathcal{U}^{\bullet}, \pi_{t} X\right) \rightarrow \check{\mathrm{H}}^{2}\left(\mathcal{U}^{\bullet}, \pi_{t+1} X\right)
$$

can be described as follows. An element $[\delta]$ of $\check{\mathrm{H}}^{0}\left(\mathcal{U}^{\bullet}, \pi_{t} X\right)$ is represented by a map

$$
\delta: S^{t} \rightarrow X_{\mathcal{U}}^{0} \bullet=\prod_{i \in I} X\left(U_{i}\right)
$$


such that $\delta_{i}$ is homotopic to $\delta_{j}$ on $V_{i j}^{\alpha}$ for every $i, j, \alpha$. Pick a specific based homotopy

$$
y_{i j}^{\alpha}: \delta_{i} \rightarrow \delta_{j}
$$

on $V_{i j}^{\alpha}$. This data determines a map $\partial \Delta_{+}^{2} \wedge \Delta_{+}^{t} \rightarrow X_{\mathcal{U}}^{2}$. such that on each face of $\Delta^{2}$, the component in $X\left(V_{i j k}^{\alpha \beta \gamma}\right)$ is one of the homotopies $y_{i j}^{\alpha}, y_{j k}^{\beta}$, or $y_{k i}^{\gamma}$. Then, let $\Lambda_{n}^{t} \subseteq \Delta^{t}$ be a horn, and let

$$
\Delta_{+}^{2} \wedge \Delta_{+}^{t} \rightarrow X_{\mathcal{U}}^{2}
$$

be a fill of the horn. Then, the restriction to

$$
\Delta_{+}^{2} \wedge \partial^{n} \Delta_{+}^{t}
$$

is in the class of $d_{2}([\delta])$.

Proof. The proof follows immediately from Construction 6.3 and Definition 5.6.

\section{Divisibility theorem}

In this section, the Čech spectral sequence is used to study the differentials $d_{2}^{\alpha}$ in the Brown-Gersten spectral sequence of twisted algebraic $K$-theory.

To a class $\alpha \in \mathrm{H}^{2}\left(U_{\text {ét }}, \mathbb{G}_{m}\right)$, one associates a stack $\mathbf{P r o j}^{\alpha}$ of $\alpha$-twisted coherent sheaves locally free and of finite rank. This is a stack of exact categories. The pointwise $K$-theory of this stack will be written $\mathbf{K}^{\alpha}$, where

$$
\mathbf{K}^{\alpha}(V)=\mathbf{K}^{Q}\left(\mathbf{P r o j}_{V}^{\alpha}\right)=B Q\left(\mathbf{P r o j}_{V}^{\alpha}\right),
$$

for $V \rightarrow U$, and where $B Q\left(\mathbf{P r o j}_{V}^{\alpha}\right)$ is the classifying space of Quillen's category $Q\left(\mathbf{P r o j}_{V}^{\alpha}\right)[\mathrm{Q}]$. The homotopy presheaves are

$$
\mathbf{K}_{k}^{\alpha}: V \mapsto \pi_{k+1} \mathbf{K}^{\alpha}(V),
$$

and the associated sheaves are $\mathcal{K}_{k}^{\alpha}$. The differentials of the Brown-Gersten spectral sequence associated to $\mathbf{K}^{\alpha}$ are written $d_{r}^{\alpha}$. The idea of an $\alpha$-twisted sheaf was apparently created by Giraud $[\mathrm{G}]$, and was revived in the thesis of Căldăraru $[\mathrm{C}]$. For a modern geometric approach, see Lieblich [L]. In the context of $K$-theory, see [A].

Let $\mathbf{K}^{\alpha \text {,ét }}=\mathbb{H K}^{\alpha}$ be a fibrant replacement for $\mathbf{K}^{\alpha}$ in the local model category of presheaves of spaces on $U_{\text {ét. }}$ When $U$ is of finite cohomological dimension, the Brown-Gersten spectral sequence of $\mathbf{K}^{\alpha}$,

$$
\mathrm{E}_{2}^{s, t}=\mathrm{H}^{s}\left(U_{\text {ét }}, \pi_{t} \mathbf{K}^{\alpha}\right)=\mathrm{H}^{s}\left(U_{\text {ét }}, \mathcal{K}_{t-1}^{\alpha}\right) \Rightarrow \pi_{t-s} \mathbf{K}^{\alpha, \text { ét }}(U),
$$

converges strongly to the homotopy of $\mathbf{K}^{\alpha, \text { ét }}$.

Definition 8.1. In $[\mathrm{A}]$, natural isomorphisms $\mathcal{K}_{k}^{\alpha} \stackrel{\simeq}{\rightrightarrows} \mathcal{K}_{k}$ are given. It follows that,

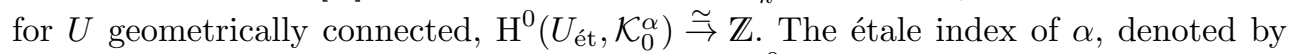
eti $(\alpha)$, is defined to be the smallest integer $n \in \mathrm{H}^{0}\left(U_{\text {ét }}, \mathcal{K}_{0}^{\alpha}\right)$ such that $d_{k}^{\alpha}(n)=0$ for all $k \geqslant 2$. In other words, eti $(\alpha)$ is the generator of

$$
\mathrm{E}_{\infty}^{0,1} \subseteq \mathrm{H}^{0}\left(U_{\text {ét }}, \mathcal{K}_{0}^{\alpha}\right)=\mathrm{H}^{0}\left(U_{\text {ét }}, \pi_{1} \mathbf{K}^{\alpha}\right) .
$$

Equivalently, eti $(\alpha)$ is the positive generator of the image of the rank map $\mathbf{K}_{0}^{\alpha \text {,ét }}(U) \rightarrow$ $\mathbb{Z}$. The period of $\alpha$, denoted by $\operatorname{per}(\alpha)$, is the order of $\alpha$ in $\mathrm{H}^{2}\left(U_{\text {ét }}, \mathbb{G}_{m}\right)$. 
Remark 8.2. In general, if $X$ is a presheaf of spaces, then the differentials leaving $\mathrm{H}^{0}\left(U, \pi_{0} X\right)$ in the Brown-Gersten spectral sequence for $X$ are all zero. This is why we use $B Q\left(\mathbf{P r o j}^{\alpha}\right)$ instead of $\Omega B Q\left(\mathbf{P r o j}^{\alpha}\right)$ as our model for $K$-theory. Of course, one can stabilize the argument and work with presheaves of spectra. But, that argument requires another level of complexity which we wished to avoid.

A key ingredient of our main theorem is the following lemma, which allows us to identify the critical lift in the description of $d_{2}$ in the Čech spectral sequence for twisted $K$-theory. First, some notation.

Let $E$ be an exact category, let $M_{i}, i=0,1,2$, be objects of $E$, and let $\theta_{i j}: M_{i} \rightarrow$ $M_{j}$ be isomorphisms for $\theta_{01}, \theta_{12}$, and $\theta_{20}$. Then, the $M_{i}$ determine loops in $B Q E$, that is, elements of $\pi_{1} B Q E$. Recall here that the base-point of $B Q E$ is the zero object of $E$. The isomorphisms $\theta_{i j}$ determine homotopies of the loops. We are thus in the position of having a map $\partial \Delta_{+}^{2} \wedge \Delta_{+}^{1} \rightarrow B Q E$. Use the horn $\Lambda_{0}^{1} \subseteq \Delta^{1}$ to create a lift $\Delta_{+}^{2} \wedge \Delta_{+}^{1} \rightarrow B Q E$. Then, $\Delta_{+}^{2} \wedge \partial^{0} \Delta_{+}^{1} \rightarrow B Q E$ is an element of $\pi_{2} B Q E$, say $\sigma$.

Lemma 8.3. The element $\sigma \in \pi_{2} B Q E$ is the same as the class of $\pi_{2} B Q E$ canonically associated to the automorphism $\theta_{20} \circ \theta_{12} \circ \theta_{01}$ of $M_{0}$.

Proof. Each face $\partial^{n} \Delta_{+}^{2} \wedge \Delta^{1}$, i.e., each homotopy $\theta_{i j}$, corresponds to map

$$
\theta_{i j}: S^{2}-\left(D^{1} \vee D^{1}\right) \rightarrow B Q E,
$$

where the restriction of $\theta_{i j}$ to the first (resp. second) boundary disc is $M_{i}$ (resp. $\left.M_{j}\right)$. Let $X=S^{2}-\left(D^{1} \vee D^{1}\right)$. Then, together, $\theta_{01}$ and $\theta_{12}$ induce a map from the connected sum of $X$ with itself glued along $M_{1}$. This connected sum is itself homotopy equivalent to $X$. Throwing $\theta_{20}$ into the picture, we get two maps $X \rightarrow B Q E$ which on one disc boundary are $M_{0}$ and on the second are $M_{2}$. Thus, they induce a map from the connected sum of $X$ with itself along the figure eight $S^{1} \vee S^{1}$. But, this connected sum is homotopy equivalent to $S^{2}$. The homotopy class of this map is $\sigma$.

Now we check that $\sigma$ agrees with the automorphism of $M_{0}$ above.

Recall that the category $Q E$ consists of the same objects as $E$ but has as morphisms from $L$ to $N$ the collection of diagrams $L \nleftarrow M \hookrightarrow N$, where $\nleftarrow$ and $\hookrightarrow$ denote admissible surjections and injections in $E$, modulo isomorphisms of such diagrams which are equalities on $L$ and $N$.

An element $M$ of $E$ gives rise to an element $r_{M}$ in $\mathbf{K}_{0}(E)$. As a based loop in $B Q E$, this is constructed as the composition of $i_{M}: 0 \nleftarrow 0 \hookrightarrow M$ with the inverse of $q_{M}: 0 \nleftarrow M \hookrightarrow M$. See [S].

As explained in [S, pp. 43-45], an exact sequence $0 \rightarrow L \hookrightarrow M \rightarrow N \rightarrow 0$ in $E$ corresponds to a choice of homotopy between $r_{M}$ and $r_{L} \cdot r_{N}$. This homotopy is constructed explicitly in ibid. as a map from the 2-sphere with a wedge sum of three discs removed, where the boundaries of these discs are the correctly oriented $r_{*}$.

In particular, given an isomorphism $\theta: L \stackrel{\widetilde{\sim}}{\rightarrow} M$, we get a map

$$
a_{\theta}: S^{2}-\left(D^{1} \vee D^{1} \vee D^{1}\right) \rightarrow B Q E
$$

with three discs removed. Since $\theta$ is an isomorphism, one of those discs can be filled in in $B Q E$, and we get a map $X \rightarrow B Q E$. So, the map from the punctured $S^{2}$ is just a proof of the equality $[L]=[M]$ in $\mathbf{K}_{0}(E)$. 
When $\theta: L \stackrel{\widetilde{\rightrightarrows}}{\rightarrow} L$, the map $a_{\theta}$ is a map from $X$ to $B Q E$ whose restrictions to the two boundary discs is the same. Thus, $a_{\theta}$ induces a map $S^{2} \rightarrow B Q E$. This is the element of $\pi_{2} B Q E$ associated to $\theta$.

In our situation of the $M_{i}$ and the $\theta_{i j}$, we have maps $a_{\theta_{i j}}: X \rightarrow B Q E$. Looking at the construction of these maps, it is easy to see that $a_{\theta_{12} \circ \theta_{01}}: X \rightarrow B Q E$ is homotopic to gluing the two maps $a_{\theta_{01}}$ and $a_{\theta_{12}}$ along their common boundary circle $M_{1}$. Similarly, gluing $a_{\theta_{20}}$ to $a_{\theta_{12} \circ \theta_{01}}$ along $M_{2}$ is homotopic to

$$
a=a_{\theta_{20} \circ \theta_{12} \circ \theta_{01}} .
$$

As identified above, since $a$ agrees on its two boundary circles, $a$ induces a map $S^{2} \rightarrow B Q E$ that is homotopic to $\sigma$. But, this map is also the map associated to the automorphism $\theta_{20} \circ \theta_{12} \circ \theta_{01}$ of $M_{0}$, so the lemma follows.

Construction 8.4. We describe how to reconstruct the class $\alpha \in \mathrm{H}^{2}\left(U_{\text {ét }}, \mathbb{G}_{m}\right)$ from the stack Proj ${ }^{\alpha}$. The rank 1 objects of $\mathbf{P r o j}^{\alpha}$ form a $\mathbb{G}_{m}$-gerbe, $\mathbf{P i c}^{\alpha}$. Recall from $[\mathrm{G}$, Section IV.3.4] or [B, Theorem 5.2.8] the following procedure. First, one takes a cover $\mathcal{U}$ of $U$ such that there is an object of $\mathcal{L}_{i} \in \mathbf{P i c}_{U_{i}}^{\alpha}$ for all $i$. Second, choose isomorphisms

$$
\sigma_{i}:\left.\operatorname{Aut}\left(\mathcal{L}_{i}\right) \stackrel{\widetilde{\sim}}{\rightarrow} \mathbb{G}_{m}\right|_{U_{i}},
$$

Third, pick isomorphisms

$$
\theta_{i j}^{\alpha}: \mathcal{L}_{i} \stackrel{\widetilde{\rightarrow}}{\rightarrow} \mathcal{L}_{j}
$$

on a suitable refinement 1-hypercover $\mathcal{V}_{A} \rightarrow \mathcal{U}_{I} \rightarrow U$. The composition

$$
\theta_{k i}^{\delta} \circ \theta_{j k}^{\beta} \circ \theta_{i j}^{\alpha}
$$

is an element of $\operatorname{Aut}\left(\mathcal{L}_{i}\right)\left(Z_{i j k}^{\alpha \beta \delta}\right)$, where

$$
Z_{i j k}^{\alpha \beta \delta}=V_{i j}^{\alpha} \times_{U_{j}} V_{j k}^{\beta} \times_{U_{k}} V_{i k}^{\delta} .
$$

Then,

$$
\sigma_{i}\left(\theta_{k i}^{\delta} \circ \theta_{j k}^{\beta} \circ \theta_{i j}^{\alpha}\right) \in \mathbb{G}_{m}\left(Z_{i j k}^{\alpha \beta \delta}\right)
$$

defines a 2-cocycle in $\mathbb{G}_{m}$ which is in the same cohomology class as $\alpha$.

Theorem 8.5. Suppose that $U$ is geometrically connected and quasi-separated. Let $\alpha \in \mathrm{H}^{2}\left(U_{\text {ét }}, \mathbb{G}_{m}\right)$. Then, $d_{2}^{\alpha}([1])=\alpha$, through the natural isomorphism

$$
\mathrm{H}^{2}\left(U_{\text {ét }}, \mathcal{K}_{1}^{\alpha}\right) \stackrel{\simeq}{\rightrightarrows} \mathrm{H}^{2}\left(U_{\text {ét }}, \mathcal{K}_{1}\right) \stackrel{\simeq}{\rightrightarrows} \mathrm{H}^{2}\left(U_{\text {ét }}, \mathbb{G}_{m}\right) .
$$

Proof. Let $\alpha \in \mathrm{H}^{2}\left(U_{\text {ét }}, \mathbb{G}_{m}\right)$ be defined by a Čech cocycle $\check{\alpha} \in \check{\mathrm{H}}^{2}\left(\mathcal{U}^{\bullet}, \mathbb{G}_{m}\right)$ for a hypercover $\mathcal{V} \rightarrow \mathcal{U} \rightarrow U$. Using Theorem 5.7, in order to prove the theorem, it suffices to prove that $d_{2}([1])=\check{\alpha}$ in the Čech approximation spectral sequence associated to $\mathcal{U}^{\bullet}$. (The existence of such a hypercover is where the quasi-separated hypothesis is used; see [AGV, Theorem V.7.4.1].) Let

$$
Z_{i j k}^{\alpha \beta \delta}=V_{i j}^{\alpha} \times_{U_{j}} V_{j k}^{\beta} \times_{U_{k}} V_{i k}^{\delta} .
$$

We may represent $[1] \in \check{\mathrm{H}}^{0}\left(\mathcal{U}^{\bullet}, \mathcal{K}_{0}^{\alpha}\right)$ by an $\alpha$-twisted line bundle $\mathcal{L}_{i}$ on each $U_{i}$ of $\mathcal{U} \bullet$. 
A homotopy from $\mathcal{L}_{i}$ to $\mathcal{L}_{j}$ is just an isomorphism

$$
\theta_{i j}^{\alpha}:\left.\left.\mathcal{L}_{i}\right|_{V_{i j}^{\alpha}} \stackrel{\sim}{\rightarrow} \mathcal{L}_{j}\right|_{V_{i j}^{\alpha}}
$$

where such an isomorphism exists, up to possibly refining the hypercover $\mathcal{U}^{\bullet}$. Then, by Theorem 7.1 and Lemma 8.3, the class of the automorphism

$$
\theta_{k i}^{\delta} \circ \theta_{j k}^{\beta} \circ \theta_{i j}^{\alpha}
$$

of $\left.\mathcal{L}_{i}\right|_{Z_{i j k}^{\alpha \beta \delta}}$ in $\mathbf{K}_{1}^{\alpha}\left(Z_{i j k}^{\alpha \beta \delta}\right)$ is the $Z_{i j k}^{\alpha \beta \delta}$-component of $d_{2}^{\alpha}([1])$.

The $\alpha$-twisted line bundles $\mathcal{L}_{i}$ on $U_{i}$ induce pointwise weak equivalences of $K$ theory presheaves $\phi_{i}:\left.\left.\mathbf{K}^{\alpha}\right|_{U_{i}} \stackrel{\sim}{\rightarrow} \mathbf{K}\right|_{U_{i}}$ by tensor product with $\mathcal{L}_{i}^{-1}$. As shown in [A], these local morphisms patch to create natural isomorphisms of $K$-theory sheaves

$$
\phi_{i}: \mathcal{K}_{k}^{\alpha} \stackrel{\sim}{\rightarrow} \mathcal{K}_{k},
$$

and of $K$-cohomology groups, in particular of

$$
\mathrm{H}^{2}\left(U_{\text {ét }}, \mathcal{K}_{1}^{\alpha}\right) \stackrel{\simeq}{\rightarrow} \mathrm{H}^{2}\left(U_{\text {ét }}, \mathcal{K}_{1}\right) .
$$

Define $\sigma_{i}$ by fixing an isomorphism

$$
\sigma_{i}:\left.\mathcal{L}_{i} \otimes \mathcal{L}_{i}^{-1} \stackrel{\widetilde{\rightrightarrows}}{\rightarrow} \mathbb{G}_{m}\right|_{U_{i}},
$$

possibly refining $\mathcal{U}^{\bullet}$. Then, the diagram

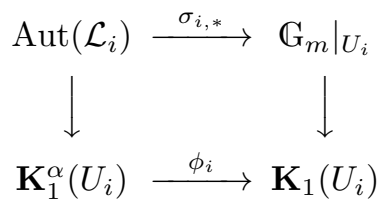

is commutative, where $\sigma_{i, *}$ is the natural isomorphism induced by $\sigma_{i}$.

The diagram and Construction 8.4 imply that $d_{2}^{\alpha}([1])$ maps to the image of $\check{\alpha}$ in the map $\check{\mathrm{H}}^{2}\left(\mathcal{U}^{\bullet}, \mathbb{G}_{m}\right) \rightarrow \check{\mathrm{H}}^{2}\left(\mathcal{U}^{\bullet}, \mathbf{K}_{1}\right)$.

Remark 8.6. There is a more sophisticated version of this theorem which uses the $K$ theory ring spectrum $\mathbf{K}$. Then the $\mathbf{K}^{\alpha}$ are module spectra over this ring spectrum. In this situation, the descent spectral sequence for $\mathbf{K}^{\alpha}$ is a module over the descent spectral sequence for $\mathbf{K}$, and thus, if $x \in \mathrm{H}^{s}\left(U_{\text {ét }}, \mathcal{K}_{t}^{\alpha}\right)$, we may write $x=1_{\alpha} \cup y$, with $y \in \mathrm{H}^{s}\left(U_{\text {ét }}, \mathcal{K}_{t}\right)$ and $1 \in \mathrm{H}^{0}\left(U_{\text {ét }}, \mathcal{K}_{0}^{\alpha}\right)$ the generator. Thus,

$$
d_{2}^{\alpha}(x)=d_{2}^{\alpha}\left(1_{\alpha}\right) \cup x \pm 1_{\alpha} \cup d_{2}(y)=\alpha \cup x \pm 1_{\alpha} \cup d_{2}(y) .
$$

So, all of the differentials in the $\mathrm{E}_{2}$-page are determined by the theorem and the differentials of the descent spectral sequence for $\mathbf{K}$.

Theorem 8.7 (Divisibility). Let $U$ be geometrically connected and quasi-separated. If $\alpha \in \mathrm{H}^{2}\left(U_{\text {ét }}, \mathbb{G}_{m}\right)$, then

$$
\operatorname{per}(\alpha) \mid \operatorname{eti}(\alpha) \text {. }
$$

Proof. This follows immediately from Theorem 8.5. Indeed, since $d_{2}^{\alpha}([1])=\alpha$, it follows that $\operatorname{per}(\alpha)$ generates $\mathrm{E}_{3}^{0,1} \subseteq \mathrm{H}^{0}\left(U_{\text {ét }}, \mathbb{Z}\right)$ in the Brown-Gersten spectral sequence. Therefore, the generator of $\mathrm{E}_{\infty}^{0,1}$ belongs to the subgroup $\operatorname{per}(\alpha) \cdot \mathbb{Z}$. In other words, $\operatorname{per}(\alpha)$ divides eti $(\alpha)$. 
Remark 8.8. In $[\mathrm{KL}]$, there is an Atiyah-Hirzebruch spectral sequence in étale motivic cohomology

$$
\mathrm{H}_{\text {ét }}^{p-q}(X, \mathbb{Z}(-q)) \Rightarrow \mathbf{K}_{-p-q}^{\mathrm{ét}}(\mathcal{A}),
$$

where $\mathcal{A}$ represents a class $\alpha \in \operatorname{Br}(X)$. Kahn and Levine show [KL, Proposition 6.9.1] that

$$
d_{2}([1])=\alpha
$$

in $\mathrm{H}_{\text {ét }}^{3}(X, \mathbb{Z}(1))=\operatorname{Br}(X)$.

Similarly, in $[\mathrm{AS}]$, in the Atiyah-Hirzebruch spectral sequence

$$
\mathrm{H}^{p}(X, \mathbb{Z}(q / 2)) \Rightarrow K U^{p-q}(X)_{\alpha}
$$

converging to twisted topological $K$-theory, it is shown [AS, Proposition 4.6] that

$$
d_{3}([1])=\alpha
$$

in $\mathrm{H}^{3}(X, \mathbb{Z})$. Note that, to be precise, the statement in the paper of Atiyah-Segal gives a spectral sequence for $\alpha$-twisted topological $K$-theory and the differential is $d_{3}([1])=-\alpha$. However, their construction of $\alpha$-twisted topological $K$-theory is in some sense dual to ours, which explains the difference in sign. This distinction is discussed in [ABG, Section 5].

\section{Index and étale index}

Let $k$ be a field of finite etale cohomological dimension. Then the main result of [A] is that

$$
\operatorname{eti}(\alpha) \mid \operatorname{per}(\alpha)^{\left[\frac{d}{2}\right]},
$$

for all $\alpha \in \operatorname{Br}(k)$ having period not divisible by a few small primes. Define $e(k)$ to be the smallest integer such that

$$
\text { eti }(\alpha) \mid \operatorname{per}(\alpha)^{e(k)}
$$

for all $\alpha \in \operatorname{Br}(k)$ whose period is not divisible by the characteristic of $k$. Such an integer exists by [A, Theorem 6.10]. Moreover, Theorem 8.7 says that $e(k) \geqslant 1$. Recall, in the notation of the introduction, the group

$$
F^{\alpha}=\mathbf{K}_{0}^{\alpha, \text { ét }}(k)^{(0)} / \mathbf{K}_{0}^{\alpha}(k)^{(0)} .
$$

Its order is $\frac{i n d(\alpha)}{\operatorname{eti}(\alpha)}$.

Definition 9.1. Say that the field $k$ has property $B_{n}$ if $F^{\alpha}$ is of order at most $\operatorname{per}(\alpha)^{n}$ for all $\alpha \in \operatorname{Br}(k)$.

Proposition 9.2. Let $k$ be a field of finite etale cohomological dimension d, and let $e(k)$ be the integer defined above. Then, the period-index conjecture holds for $k$ if $k$ has property $B_{d-1-e(k)}$. If the period-index conjecture holds for $k$, then $k$ has property $B_{d-2}$. 
Proof. Fix $\alpha \in \operatorname{Br}(k)$. Then, eti $(\alpha) \mid \operatorname{per}(\alpha)^{e(k)}$. If the order of $F^{\alpha}$ is at most

$$
\operatorname{per}(\alpha)^{d-1-e(k)},
$$

then,

$$
\operatorname{ind}(\alpha) \mid e t i(\alpha)^{d-1-e(k)},
$$

whence the first statement. To prove the second statement, it suffices to note that

$$
\frac{i n d(\alpha)}{\operatorname{eti}(\alpha)} \leqslant \frac{\operatorname{per}(\alpha)^{d-1}}{\operatorname{per}(\alpha)},
$$

by the period-index conjecture and Theorem 8.7 .

Question 9.3. If the period-index conjecture holds for $k$ does it imply that $k$ has property $B_{d-1-e(k)}$ ?

Remark 9.4. The proposition is interesting because it reveals a connection between the period-index conjecture and étale descent for (twisted) $K$-theory.

Remark 9.5. We do not know the integer $e(k)$ for any fields besides 2-dimensional fields where the period-index conjecture is known, in which case $e(k)=1$. Our guess is that it is as big as possible, namely $\left[\frac{d}{2}\right]$. Some evidence for this may be that the fields such as $k=\mathbb{C}\left(\left(t_{1}\right)\right) \cdots\left(\left(t_{d}\right)\right)$ have $\operatorname{ind}(\alpha) \mid \operatorname{per}(\alpha)^{\left[\frac{d}{2}\right]}$, so it is natural to ask whether eti $(\alpha)=\operatorname{ind}(\alpha)$ for these fields. This is a line of inquiry we are currently pursuing.

\section{References}

[ABG] Matthew Ando, Andrew J. Blumberg, and David Gepner, Twists of K-theory and TMF, Superstrings, geometry, topology, and $C^{*}$-algebras, Proc. Sympos. Pure Math., vol. 81, Amer. Math. Soc., Providence, RI, 2010, pp. 27-63.

[A] Benjamin Antieau, Cohomological obstruction theory for Brauer classes and the period-index problem. J. K-Theory, to appear.

[AGV] M. Artin, A. Grothendieck, and J. L. Verdier (eds.), Théorie des topos et cohomologie étale des schémas. Tome 2, Lecture Notes in Mathematics, vol. 270, Springer-Verlag, Berlin, 1972. Séminaire de Géométrie Algébrique du Bois-Marie 1963-1964 (SGA 4), Dirigé par M. Artin, A. Grothendieck et J. L. Verdier. Avec la collaboration de N. Bourbaki, P. Deligne et B. Saint-Donat.

[AS] Michael Atiyah and Graeme Segal, Twisted K-theory and cohomology, Inspired by S. S. Chern, 2006, pp. 5-43.

[BK] A. K. Bousfield and D. M. Kan, Homotopy limits, completions and localizations, Lecture Notes in Mathematics, vol. 304, Springer-Verlag, Berlin, 1972.

[B] Jean-Luc Brylinski, Loop spaces, characteristic classes and geometric quantization, Progress in Mathematics, vol. 107, Birkhäuser Boston Inc., Boston, MA, 1993.

[CT] J.-L. Colliot-Thélène, Die Brauersche Gruppe ; ihre Verallgemeinerungen und Anwendungen in der arithmetischen Geometrie, 2001. Unpublished lecture notes.

[C] Andrei Căldăraru, Derived categories of twisted sheaves on Calabi-Yau manifolds, Ph.D. Thesis, 2000.

[DHI] Daniel Dugger, Sharon Hollander, and Daniel C. Isaksen, Hypercovers and simplicial presheaves, Math. Proc. Cambridge Philos. Soc. 136 (2004), no. 1, 9-51.

[GS] H. Gillet and C. Soulé, Filtrations on higher algebraic K-theory, Algebraic K-theory (Seattle, WA, 1997), 1999, pp. 89-148.

[G] Jean Giraud, Cohomologie non abélienne, Springer-Verlag, Berlin, 1971. Die Grundlehren der mathematischen Wissenschaften, Band 179.

[GJ] Paul G. Goerss and John F. Jardine, Simplicial homotopy theory, Progress in Mathematics, vol. 174, Birkhäuser Verlag, Basel, 1999. 
[J] J. F. Jardine, Simplicial presheaves, J. Pure Appl. Algebra 47 (1987), no. 1, 35-87.

[KL] Bruno Kahn and Marc Levine, Motives of Azumaya algebras, J. Inst. Math. Jussieu 9 (2010), no. 3, 481-599.

[L] Max Lieblich, Twisted sheaves and the period-index problem, Compos. Math. 144 (2008), no. 1, 1-31.

[Q] Daniel Quillen, Higher algebraic K-theory. I, Algebraic $K$-theory, I: Higher $K$-theories (Proc. Conf., Battelle Memorial Inst., Seattle, Wash., 1972), 1973, pp. 85-147.

[S] V. Srinivas, Algebraic K-theory, Second, Progress in Mathematics, vol. 90, Birkhäuser Boston Inc., Boston, MA, 1996

[T] Robert W. Thomason, Algebraic K-theory and étale cohomology, Ann. Sci. École Norm. Sup. (4) 18 (1985), no. 3, 437-552.

Benjamin Antieau antieau@math.ucla.edu

Department of Mathematics, University of California Los Angeles, Box 951555, Los Angeles, CA 90095-1555, USA 